Алгебра и анализ

Том 20 (2008), № 3
St. Petersburg Math. J.

Vol. 20 (2009), No. 3, Pages 407-445

S 1061-0022(09)01054-1

Article electronically published on April 7, 2009

\title{
FESENKO RECIPROCITY MAP
}

\author{
K. I. IKEDA AND E. SERBEST
}

Dedicated to our teacher Mehpare Bilhan

\begin{abstract}
In recent papers, Fesenko has defined the non-Abelian local reciprocity map for every totally ramified arithmetically profinite (APF) Galois extension of a given local field $K$, by extending the work of Hazewinkel and Neukirch-Iwasawa. The theory of Fesenko extends the previous non-Abelian generalizations of local class field theory given by Koch-de Shalit and by A. Gurevich. In this paper, which is research-expository in nature, we give a detailed account of Fesenko's work, including all the skipped proofs.
\end{abstract}

In a series of very interesting papers [1, 2, 3], Fesenko defined the non-Abelian local reciprocity map for every totally ramified arithmetically profinite (APF) Galois extension of a given local field $K$ by extending the work of Hazewinkel [8] and Neukirch-Iwasawa [15]. "Fesenko theory" extends the previous non-Abelian generalizations of local class field theory given by Koch and de Shalit in [13] and by A. Gurevich in [7].

In this paper, which is research-expository in nature, we give a very detailed account of Fesenko's work 1, 2, 3, thereby complementing those papers by including all the proofs. Let us describe how our paper is organized. In the first Section, we briefly review the Abelian local class field theory and the construction of the local Artin reciprocity map, following the Hazewinkel method and the Neukirch-Iwasawa method. In Sections 3 and 4, we follow [4, 5, 6, and 17] to review the theory of APF extensions over $K$, and sketch the construction of Fontaine-Wintenberger's field of norms $\mathbb{X}(L / K)$ attached to an APF extension $L / K$. In order to do so, we briefly review the ramification theory of $K$ in Section 2. Finally, in Section 5, we give a detailed construction of the Fesenko reciprocity map $\Phi_{L / K}^{(\varphi)}$ defined for any totally ramified and APF Galois extension $L$ over $K$ under the assumption that $\boldsymbol{\mu}_{p}\left(K^{\mathrm{sep}}\right) \subset K$, where $p=\operatorname{char}\left(\kappa_{K}\right)$, and investigate the functorial and ramification-theoretic properties of the Fesenko reciprocity maps defined for totally ramified and APF Galois extensions over $K$.

In a related paper (see [10]), we shall extend Fesenko's construction to any Galois extension of $K$ (in a fixed $K^{\mathrm{sep}}$ ), and construct the non-Abelian local class field theory. Thus, we feel that the present paper together with [1, 2, 3] should be viewed as the technical and theoretical background, an introduction, as well as an appendix to the paper [10] on "generalized Fesenko theory". A similar theory was announced by Laubie in [14, which is an extension of the paper 13 by Koch and de Shalit. The relationship of the Laubie theory with our generalized Fesenko theory will also be investigated in [10].

Notation. Throughout this work, $K$ denotes a local field (a complete discrete valuation field) with finite residue field $O_{K} / \mathfrak{p}_{K}=: \kappa_{K}$ of $q_{K}=q=p^{f}$ elements with $p$ a prime

2000 Mathematics Subject Classification. Primary 11S20, 11S31.

Key words and phrases. Local fields, higher-ramification theory, APF extensions, Fontaine-Wintenberger field of norms, Fesenko reciprocity map, non-Abelian local class field theory, $p$-adic local Langlands correspondence. 
number, where $O_{K}$ denotes the ring of integers in $K$ with a unique maximal ideal $\mathfrak{p}_{K}$. Let $\boldsymbol{\nu}_{K}$ denote the corresponding normalized valuation on $K$ (normalized by $\boldsymbol{\nu}_{K}\left(K^{\times}\right)=\mathbb{Z}$ ), and let $\widetilde{\boldsymbol{\nu}}$ be a unique extension of $\boldsymbol{\nu}_{K}$ to a fixed separable closure $K^{\text {sep }}$ of $K$. For any subextension $L / K$ of $K^{\mathrm{sep}} / K$, the normalized form of the valuation $\left.\widetilde{\boldsymbol{\nu}}\right|_{L}$ on $L$ will be denoted by $\boldsymbol{\nu}_{L}$. Finally, we let $G_{K}$ denote the absolute Galois group $\operatorname{Gal}\left(K^{\mathrm{sep}} / K\right)$.

\section{§1. Abelian local Class Field THEORY}

Let $K$ be a local field. We fix a separable closure $K^{\text {sep }}$ of $K$. Let $G_{K}$ denote the absolute Galois group Gal $\left(K^{\mathrm{sep}} / K\right)$ of $K$. By the construction of absolute Galois groups, $G_{K}$ is a profinite topological group with respect to the Krull topology. Now, let $G_{K}^{a b}$ denote the maximal Abelian Hausdorff quotient group $G_{K} / G_{K}^{\prime}$ of the topological group $G_{K}$, where $G_{K}^{\prime}$ denotes the closure of the first commutator subgroup $\left[G_{K}, G_{K}\right]$ of $G_{K}$.

Recall that the Abelian local class field theory for the local field $K$ establishes a unique natural algebraic and topological isomorphism

$$
\alpha_{K}: \widehat{K^{\times}} \stackrel{\sim}{\longrightarrow} G_{K}^{a b},
$$

where the topological group $\widehat{K^{\times}}$is the profinite completion of the multiplicative group $K^{\times}$, satisfying the following conditions.

(1) Let $W_{K}$ denote the Weil group of $K$. Then

$$
\alpha_{K}\left(K^{\times}\right)=W_{K}^{a b} .
$$

(2) For every Abelian extension $L / K$ (always assumed to be a subextension of $K^{\text {sep }} / K$, where a separable closure $K^{\text {sep }}$ of $K$ is fixed throughout the remainder of the text), the surjective and continuous homomorphism

$$
\alpha_{L / K}: \widehat{K^{\times}} \stackrel{\alpha_{K}}{\longrightarrow} G_{K}^{a b} \stackrel{\text { res }_{L}}{\longrightarrow} \operatorname{Gal}(L / K)
$$

satisfies

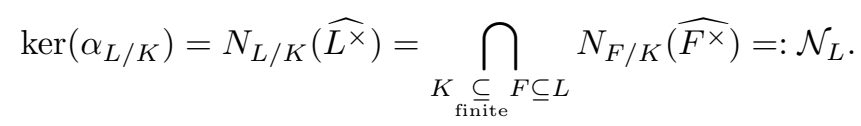

(3) For each Abelian extension $L / K$, the mapping

$$
L \mapsto \mathcal{N}_{L}
$$

determines a bijective correspondence

$$
\{L / K: \text { Abelian }\} \leftrightarrow\left\{\mathcal{N}: \mathcal{N} \underset{\text { closed }}{\leq} \widehat{K^{\times}}\right\}
$$

that satisfies the following conditions: for every Abelian extension $L, L_{1}$, and $L_{2}$ over $K$,

(i) $L / K$ is a finite extension if and only if

$$
\mathcal{N}_{L} \underset{\text { open }}{\leq} \widehat{K^{\times}}
$$

(this is equivalent to $\left.\left(\widehat{K^{\times}}: \mathcal{N}_{L}\right)<\infty\right)$;

(ii) $L_{1} \subseteq L_{2} \Leftrightarrow \mathcal{N}_{L_{1}} \supseteq \mathcal{N}_{L_{2}}$;

(iii) $\mathcal{N}_{L_{1} L_{2}}=\mathcal{N}_{L_{1}} \cap \mathcal{N}_{L_{2}}$;

(iv) $\mathcal{N}_{L_{1} \cap L_{2}}=\mathcal{N}_{L_{1}} \mathcal{N}_{L_{2}}$. 
(4) (Ramification theory) 11 Let $L / K$ be an Abelian extension. For every integer $0 \leq i \in \mathbb{Z}$ and every real number $\nu \in(i-1, i]$,

$$
x \in U_{K}^{i} \mathcal{N}_{L} \Leftrightarrow \alpha_{L / K}(x) \in \operatorname{Gal}(L / K)^{\nu},
$$

where $x \in \widehat{K^{\times}}$.

(5) (Functoriality). Let $L / K$ be an Abelian extension.

(i) For $\gamma \in \operatorname{Aut}(K)$,

$$
\alpha_{K}(\gamma(x))=\widetilde{\gamma} \alpha_{K}(x) \widetilde{\gamma}^{-1}
$$

for every $x \in \widehat{K^{\times}}$, where $\widetilde{\gamma}: K^{a b} \rightarrow K^{a b}$ is any automorphism of the field $K^{a b}$ satisfying $\left.\widetilde{\gamma}\right|_{K}=\gamma$;

(ii) under the condition $[L: K]<\infty$,

$$
\left.\alpha_{L}(x)\right|_{K^{a b}}=\alpha_{K}\left(N_{L / K}(x)\right)
$$

for every $x \in \widehat{L^{\times}}$;

(iii) under the condition $[L: K]<\infty$,

$$
\alpha_{L}(x)=V_{K \rightarrow L}\left(\alpha_{K}(x)\right)
$$

for every $x \in \widehat{K^{\times}}$, where $V_{K \rightarrow L}: G_{K}^{a b} \rightarrow G_{L}^{a b}$ is the group-theoretic transfer homomorphism (Verlagerung).

This unique algebraic and topological isomorphism $\alpha_{K}: \widehat{K^{\times}} \rightarrow G_{K}^{a b}$ is called the local Artin reciprocity map of $K$.

There are many constructions of the local Artin reciprocity map of $K$, including the cohomological and analytical constructions. Now, in the remaining part of this section, we shall review the construction of the local Artin reciprocity map $\alpha_{K}: \widehat{K^{\times}} \rightarrow G_{K}^{a b}$ of $K$, following Hazewinkel 8 and Iwasawa-Neukirch [12, 15. As usual, let $K^{n r}$ denote the maximal unramified extension of $K$. It is well known that $K^{n r}$ is not a complete field with respect to the valuation $\nu_{K^{n r}}$ on $K^{n r}$ induced from the valuation $\nu_{K}$ of $K$. Let $\widetilde{K}$ denote the completion of $K^{n r}$ with respect to the valuation $\nu_{K^{n r}}$ on $K^{n r}$. For a Galois extension $L / K$, put $L^{n r}=L K^{n r}$ and $\widetilde{L}=L \widetilde{K}$. For each $\tau \in \operatorname{Gal}(L / K)$, we choose $\tau^{*} \in \operatorname{Gal}\left(L^{n r} / K\right)$ in such a way that

(1) $\left.\tau^{*}\right|_{L}=\tau$

(2) $\left.\tau^{*}\right|_{K^{n r}}=\varphi^{n}$ for some $0<n \in \mathbb{Z}$, where $\varphi \in \operatorname{Gal}\left(K^{n r} / K\right)$ denotes the (arithmetic) Frobenius automorphism of $K$.

The fixed field $\left(L^{n r} \tau^{\tau^{*}}=\left\{x \in L^{n r}: \tau^{*}(x)=x\right\}\right.$ of this chosen $\tau^{*} \in \operatorname{Gal}\left(L^{n r} / K\right)$ in $L^{n r}$ will be denoted by $\Sigma_{\tau^{*}}$; we have $\left[\Sigma_{\tau^{*}}: K\right]<\infty$.

The Iwasawa-Neukirch mapping

$$
\iota_{L / K}: \operatorname{Gal}(L / K) \rightarrow K^{\times} / N_{L / K}\left(L^{\times}\right)
$$

is then defined by

$$
\iota_{L / K}: \tau \mapsto N_{\Sigma_{\tau^{*} / K}}\left(\pi_{\Sigma_{\tau^{*}}}\right) \quad \bmod N_{L / K}\left(L^{\times}\right)
$$

for every $\tau \in \operatorname{Gal}(L / K)$, where $\pi_{\Sigma_{\tau^{*}}}$ denotes any prime element of $\Sigma_{\tau^{*}}$.

Suppose now that, moreover, the Galois extension $L / K$ is a totally ramified and finite extension. We introduce a subgroup $\widetilde{V}(L / K)$ in the unit group $U_{\widetilde{L}}=O_{\widetilde{L}}^{\times}$of the ring of integers $O_{\widetilde{L}}$ of the local field $\widetilde{L}$ by

$$
\widetilde{V}(L / K)=\left\langle u^{\sigma-1}: u \in U_{\widetilde{L}}, \sigma \in \operatorname{Gal}(L / K)\right\rangle .
$$

\footnotetext{
${ }^{1}$ We shall review the higher-ramification subgroups $\operatorname{Gal}(L / K)^{\nu}$ of $\operatorname{Gal}(L / K)$ (with the upper numbering) in the next section.
} 
Then the homomorphism

$$
\theta: \operatorname{Gal}(L / K) \rightarrow U_{\widetilde{L}} / \widetilde{V}(L / K)
$$

defined by

$$
\theta: \sigma \mapsto \frac{\sigma\left(\pi_{L}\right)}{\pi_{L}} \bmod \widetilde{V}(L / K)
$$

for every $\sigma \in \operatorname{Gal}(L / K)$, makes the triangle

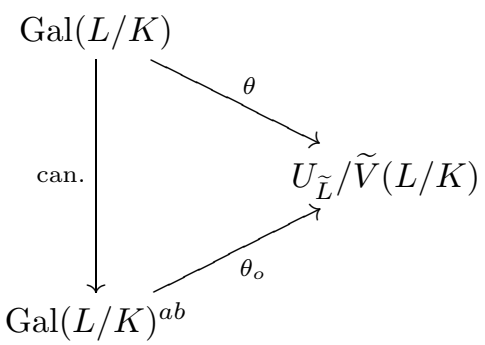

commutative. The quotient $U_{\widetilde{L}} / \widetilde{V}(L / K)$ sits in the Serre short exact sequence

$$
1 \rightarrow \operatorname{Gal}(L / K)^{a b} \stackrel{\theta_{o}}{\longrightarrow} U_{\widetilde{L}} / \widetilde{V}(L / K) \stackrel{N_{\tilde{L} / \widetilde{K}}}{\longrightarrow} U_{\widetilde{K}} \rightarrow 1 .
$$

Let $V(L / K)$ denote the subgroup in the unit group $U_{L^{n r}}$ of the ring of integers $O_{L^{n r}}$ of the maximal unramified extension $L^{n r}$ of the local field $L$ defined by

$$
V(L / K)=\left\langle u^{\sigma-1}: u \in U_{L^{n r}}, \sigma \in \operatorname{Gal}(L / K)\right\rangle .
$$

The quotient $U_{L^{n r}} / V(L / K)$ sits in the Serre short exact sequence

$$
1 \rightarrow \operatorname{Gal}(L / K)^{a b} \stackrel{\theta_{o}}{\longrightarrow} U_{L^{n r}} / V(L / K) \stackrel{N_{L^{n r} / K^{n r}}}{\longrightarrow} U_{K^{n r}} \rightarrow 1 .
$$

As before, let $\varphi \in \operatorname{Gal}\left(K^{n r} / K\right)$ denote the Frobenius automorphism of $K$. We fix any extension of the automorphism $\varphi$ of $K^{n r}$ to an automorphism of $L^{n r}$, denoted again by $\varphi$. Now, for any $u \in U_{K}$, there exists $v_{u} \in U_{L^{n r}}$ such that $u=N_{L^{n r} / K^{n r}}\left(v_{u}\right)$. Then the relation

$$
N_{L^{n r} / K^{n r}}\left(\varphi\left(v_{u}\right)\right)=\varphi\left(N_{L^{n r} / K^{n r}}\left(v_{u}\right)\right)=\varphi(u)=u,
$$

combined with the Serre short exact sequence, yields the existence of $\sigma_{u} \in \operatorname{Gal}(L / K)^{a b}$ satisfying

The Hazewinkel mapping

$$
\theta_{o}\left(\sigma_{u}\right)=\frac{\sigma_{u}\left(\pi_{L}\right)}{\pi_{L}}=\frac{v_{u}}{\varphi\left(v_{u}\right)}
$$

$$
h_{L / K}: U_{K} / N_{L / K} U_{L} \rightarrow \operatorname{Gal}(L / K)^{a b}
$$

is then defined by

for every $u \in U_{K}$.

$$
h_{L / K}: u \mapsto \sigma_{u}
$$

It turns out that if $L / K$ is a totally ramified and finite Galois extension, then the Hazewinkel mapping $h_{L / K}: U_{K} / N_{L / K} U_{L} \rightarrow \operatorname{Gal}(L / K)^{a b}$ and the Iwasawa-Neukirch mapping $\iota_{L / K}: \operatorname{Gal}(L / K) \rightarrow K^{\times} / N_{L / K}\left(L^{\times}\right)$are mutually inverse. Thus, by the uniqueness of the local Artin reciprocity map $\alpha_{K}: \widehat{K^{\times}} \rightarrow G_{K}^{a b}$ of the local field $K$, it follows that the Hazewinkel map, the Iwasawa-Neukirch map, and the local Artin map are related to each other as follows:

and

$$
h_{L / K}=\alpha_{L / K}
$$

$$
\iota_{L / K}=\alpha_{L / K}^{-1} .
$$




\section{§2. REVIEW OF RAMIFICATION THEORY}

In this section, we shall review the higher-ramification subgroups with the upper numbering of the absolute Galois group $G_{K}$ of the local field $K$, which is necessary in the theory of APF extensions over $K$. The main reference that we follow for this section is 16 .

For a finite separable extension $L / K$ and any $\sigma \in \operatorname{Hom}_{K}\left(L, K^{\text {sep }}\right)$, we introduce

$$
i_{L / K}(\sigma):=\min _{x \in O_{L}}\left\{\boldsymbol{\nu}_{L}(\sigma(x)-x)\right\}
$$

put

$$
\gamma_{t}:=\#\left\{\sigma \in \operatorname{Hom}_{K}\left(L, K^{\mathrm{sep}}\right): i_{L / K}(\sigma) \geq t+1\right\}
$$

for $-1 \leq t \in \mathbb{R}$, and define a function $\varphi_{L / K}: \mathbb{R}_{\geq-1} \rightarrow \mathbb{R}_{\geq-1}$, the Hasse-Herbrand transition function of the extension $L / K$, by

$$
\varphi_{L / K}(u):= \begin{cases}\int_{0}^{u} \frac{\gamma_{t}}{\gamma_{0}} d t & \text { if } \quad 0 \leq u \in \mathbb{R}, \\ u & \text { if }-1 \leq u \leq 0 .\end{cases}
$$

It is well known that $\varphi_{L / K}: \mathbb{R}_{\geq-1} \rightarrow \mathbb{R}_{\geq-1}$ is a continuous, monotone increasing, piecewise linear function, and induces a homeomorphism $\mathbb{R}_{\geq-1} \stackrel{\approx}{\rightarrow} \mathbb{R}_{\geq-1}$. Now, let $\psi_{L / K}: \mathbb{R}_{\geq-1} \rightarrow \mathbb{R}_{\geq-1}$ be the mapping inverse to the function $\varphi_{L / K}: \mathbb{R}_{\geq-1} \rightarrow \mathbb{R}_{\geq-1}$.

Assume that $L$ is a finite Galois extension over $K$ with Galois group $\operatorname{Gal}(L / K)=: G$. The normal subgroup $G_{u}$ of $G$ defined by

$$
G_{u}=\left\{\sigma \in G: i_{L / K}(\sigma) \geq u+1\right\}
$$

for $-1 \leq u \in \mathbb{R}$ is called the $u$ th ramification group of $G$ with the lower numbering, and has order $\gamma_{u}$. Note that $G_{u^{\prime}} \subseteq G_{u}$ for every pair $-1 \leq u, u^{\prime} \in \mathbb{R}$ satisfying $u \leq u^{\prime}$. The family $\left\{G_{u}\right\}_{u \in \mathbb{R}>-1}$ induces a filtration on $G$, called the lower ramification filtration of $G$. A break in the lower ramification filtration $\left\{G_{u}\right\}_{u \in \mathbb{R}_{>-1}}$ of $G$ is defined to be any number $u \in \mathbb{R}_{\geq-1}$ satisfying $G_{u} \neq G_{u+\varepsilon}$ for every $0<\varepsilon \in \mathbb{R}$. The function $\psi_{L / K}=\varphi_{L / K}^{-1}: \mathbb{R}_{\geq-1} \rightarrow \mathbb{R}_{\geq-1}$ induces the upper ramification filtration $\left\{G^{v}\right\}_{v \in \mathbb{R}_{\geq-1}}$ on $G$ by setting

or equivalently, by setting

$$
G^{v}:=G_{\psi_{L / K}(v)}
$$

$$
G^{\varphi_{L / K}(u)}=G_{u}
$$

for $-1 \leq v, u \in \mathbb{R}$, where $G^{v}$ is called the $v$ th upper ramification group of $G$. A break in the upper filtration $\left\{G^{v}\right\}_{v \in \mathbb{R}_{\geq-1}}$ of $G$ is defined to be any number $v \in \mathbb{R}_{\geq-1}$ satisfying $G^{v} \neq G^{v+\varepsilon}$ for every $0<\varepsilon \in \mathbb{R}$.

Remark 2.1. We mention the basic properties of lower and upper ramification filtrations on $G$. In what follows, $F / K$ denotes a subextension of $L / K$ and $H$ denotes the Galois group $\operatorname{Gal}(L / F)$ corresponding to the extension $L / F$.

(i) The lower numbering on $G$ passes well to the subgroup $H$ of $G$ in the sense that

$$
H_{u}=H \cap G_{u}
$$

for $-1 \leq u \in \mathbb{R}$

(ii) and if, furthermore, $H \triangleleft G$, then the upper numbering on $G$ passes well to the quotient $G / H$ :

$$
(G / H)^{v}=G^{v} H / H
$$

for $-1 \leq v \in \mathbb{R}$. 
(iii) The Hasse-Herbrand function and its inverse satisfy the transitive law

$$
\varphi_{L / K}=\varphi_{F / K} \circ \varphi_{L / F}
$$

and

$$
\psi_{L / K}=\psi_{L / F} \circ \psi_{F / K}
$$

If $L / K$ is an infinite Galois extension with Galois group $\operatorname{Gal}(L / K)=G$, which is a topological group under the respective Krull topology, we define the upper ramification filtration $\left\{G^{v}\right\}_{v \in \mathbb{R}_{\geq-1}}$ on $G$ by the projective limit

$$
G^{v}:=\lim _{K \subseteq \underline{\subseteq} \subset L} \operatorname{Gal}(F / K)^{v}
$$

defined over the transition morphisms $t_{F}^{F^{\prime}}(v): \operatorname{Gal}\left(F^{\prime} / K\right)^{v} \rightarrow \operatorname{Gal}(F / K)^{v}$, which are essentially the restriction morphisms from $F^{\prime}$ to $F$, defined naturally by the diagram

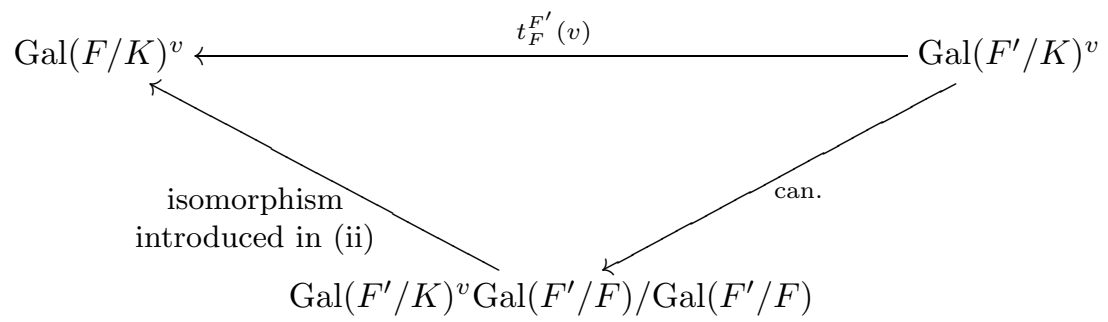

induced from (ii), as $K \subseteq F \subseteq F^{\prime} \subseteq L$ runs over all finite Galois extensions $F$ and $F^{\prime}$ over $K$ inside $L$. The topological subgroup $G^{v}$ of $G$ is called the $v$ th ramification group of $G$ in the upper numbering. Note that $G^{v^{\prime}} \subseteq G^{v}$ for every pair $-1 \leq v, v^{\prime} \in \mathbb{R}$ satisfying $v \leq v^{\prime}$, via the commutativity of the square

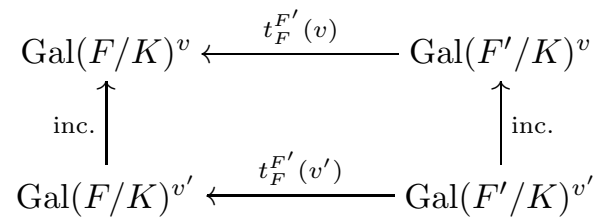

for every chain $K \subseteq F \subseteq F^{\prime} \subseteq L$ of finite Galois extensions $F$ and $F^{\prime}$ over $K$ inside $L$. Observe that

(iv) $G^{-1}=G$ and $G^{0}$ is the inertia subgroup of $G$;

(v) $\bigcap_{v \in \mathbb{R}_{\geq-1}} G^{v}=\left\langle 1_{G}\right\rangle$;

(vi) $G^{v}$ is a closed subgroup of $G$, with respect to the Krull topology, for $-1 \leq v \in \mathbb{R}$.

In this setting, a number $-1 \leq v \in \mathbb{R}$ is said to be a break in the upper ramification filtration $\left\{G^{v}\right\}_{v \in \mathbb{R}_{\geq-1}}$ of $G$ if $v$ is a break in the upper filtration of some finite quotient $G / H$ for some $H \triangleleft G$. Let $\mathcal{B}_{L / K}$ denote the set of all numbers $v \in \mathbb{R}_{\geq-1}$ that occur as breaks in the upper ramification filtration of $G$. Then:

(vii) (Hasse-Arf theorem) $\mathcal{B}_{K^{a b} / K} \subseteq \mathbb{Z} \cap \mathbb{R}_{\geq-1}$;

(viii) $\mathcal{B}_{K^{\mathrm{sep}} / K} \subseteq \mathbb{Q} \cap \mathbb{R}_{\geq-1}$.

\section{§3. APF EXTEnsions OVER $K$}

In this section, we shall briefly review a very important class of algebraic extensions over a local field $K$, called the APF extensions and introduced by Fontaine and Wintenberger (cf. [5, 6] and [17]). As in the previous section, let $\left\{G_{K}^{v}\right\}_{v \in \mathbb{R}_{\geq-1}}$ denote the upper ramification filtration of the absolute Galois group $G_{K}$ of $K$, and let $R^{v}$ denote 
the fixed field $\left(K^{\text {sep }}\right)^{G_{K}^{v}}$ of the $v$ th upper ramification subgroup $G_{K}^{v}$ of $G_{K}$ in $K^{\text {sep }}$ for $-1 \leq v \in \mathbb{R}$.

Definition 3.1. An extension $L / K$ is called an $A P F$ extension ( $A P F$ is an abbreviation for "arithmétiquement profinie") if one of the following equivalent conditions is satisfied:

(i) $G_{K}^{v} G_{L}$ is open in $G_{K}$ for every $-1 \leq v \in \mathbb{R}$;

(ii) $\left(G_{K}: G_{K}^{v} G_{L}\right)<\infty$ for every $-1 \leq v \in \mathbb{R}$;

(iii) $L \cap R^{v}$ is a finite extension over $K$ for every $-1 \leq v \in \mathbb{R}$.

Note that if $L / K$ is an APF extension, then $\left[\kappa_{L}: \kappa_{K}\right]<\infty$.

Now, let $L / K$ be an APF extension. We set $G_{L}^{0}=G_{L} \cap G_{K}^{0}$ and define

$$
\varphi_{L / K}(v)= \begin{cases}\int_{0}^{v}\left(G_{K}^{0}: G_{L}^{0} G_{K}^{x}\right) d x & \text { if } \quad 0 \leq v \in \mathbb{R}, \\ v & \text { if } \quad-1 \leq v \leq 0 .\end{cases}
$$

Then the map $v \mapsto \varphi_{L / K}(v)$ for $v \in \mathbb{R}_{\geq-1}$, which is well defined for the APF extension $L / K$, determines a continuous, strictly monotone increasing and piecewise-linear bijection $\varphi_{L / K}: \mathbb{R}_{\geq-1} \rightarrow \mathbb{R}_{\geq-1}$. We denote the inverse of this mapping by $\psi_{L / K}:=\varphi_{L / K}^{-1}$ : $\mathbb{R}_{\geq-1} \rightarrow \mathbb{R}_{\geq-1}$.

Thus, if $L / K$ is a (not necessarily finite) APF Galois extension, then we can define the higher ramification subgroups with the lower numbering $\operatorname{Gal}(L / K)_{u}$ of $\operatorname{Gal}(L / K)$, for $-1 \leq u \in \mathbb{R}$, by setting

$$
\operatorname{Gal}(L / K)_{u}:=\operatorname{Gal}(L / K)^{\varphi_{L / K}(u)} .
$$

Remark 3.2. The following should be noted:

(i) in case $L / K$ is a finite separable extension, which is clearly an APF extension by Definition 3.1, the function $\varphi_{L / K}: \mathbb{R}_{\geq-1} \rightarrow \mathbb{R}_{\geq-1}$ coincides with the HasseHerbrand transition function of $L / K$ introduced in the previous section;

(ii) if $L / K$ is a finite separable extension and $L^{\prime} / L$ is an APF extension, then $L^{\prime} / K$ is an APF extension, and the transitivity rules for the functions $\varphi_{L^{\prime} / K}, \psi_{L^{\prime} / K}$ : $\mathbb{R}_{\geq-1} \rightarrow \mathbb{R}_{\geq-1}$ hold by

$$
\varphi_{L^{\prime} / K}=\varphi_{L / K} \circ \varphi_{L^{\prime} / L}
$$

and by

$$
\psi_{L^{\prime} / K}=\psi_{L^{\prime} / L} \circ \psi_{L / K} .
$$

The next result will be extremely useful.

Lemma 3.3. Suppose that $K \subseteq F \subseteq L \subseteq K^{\text {sep }}$ is a tower of field extensions in $K^{\text {sep }}$ over $K$. Then:

(i) if $[F: K]<\infty$, then $L / K$ is an APF extension if and only if $L / F$ is an $A P F$ extension;

(ii) if $[L: F]<\infty$, then $L / K$ is an $A P F$ extension if and only if $F / K$ is an $A P F$ extension;

(iii) if $L / K$ is an $A P F$ extension, then $F / K$ is an $A P F$ extension.

Proof. For a proof, look at Proposition 1.2.3 in [17.

\section{§4. Fontaine-Wintenberger fields of nORMS}

Let $L / K$ be an infinite $A P F$ extension. For $0 \leq i \in \mathbb{Z}$, let $L_{i}$ be an increasing directed family of subextensions in $L / K$ such that

(i) $\left[L_{i}: K\right]<\infty$ for every $0 \leq i \in \mathbb{Z}$;

(ii) $\bigcup_{0 \leq i \in \mathbb{Z}} L_{i}=L$. 
Let

$$
\mathbb{X}(L / K)^{\times}={\underset{\varliminf}{\leftarrow}}_{i} L_{i}^{\times}
$$

be the projective limit of the multiplicative groups $L_{i}^{\times}$with respect to the norm homomorphisms

for every $0 \leq i, i^{\prime} \in \mathbb{Z}$ with $i \leq i^{\prime}$.

$$
N_{L_{i^{\prime}} / L_{i}}: L_{i^{\prime}}^{\times} \rightarrow L_{i}^{\times},
$$

Remark 4.1. The group $\mathbb{X}(L / K)^{\times}$does not depend on the choice of the increasing directed family of subextensions $\left\{L_{i}\right\}_{0 \leq i \in \mathbb{Z}}$ in $L / K$ satisfying conditions (i) and (ii). Thus,

$$
\mathbb{X}(L / K)^{\times}=\lim _{M \in S_{L / K}} M^{\times},
$$

where $S_{L / K}$ is the partially ordered family of all finite subextensions in $L / K$, and the projective limit is with respect to the norm

$$
N_{M_{2} / M_{1}}: M_{2}^{\times} \rightarrow M_{1}^{\times},
$$

for every $M_{1}, M_{2} \in S_{L / K}$ with $M_{1} \subseteq M_{2}$.

We put

$$
\mathbb{X}(L / K)=\mathbb{X}(L / K)^{\times} \cup\{0\},
$$

where 0 is a fixed symbol, and define the addition

$$
+: \mathbb{X}(L / K) \times \mathbb{X}(L / K) \rightarrow \mathbb{X}(L / K)
$$

by the rule

$$
\left(\alpha_{M}\right)+\left(\beta_{M}\right)=\left(\gamma_{M}\right),
$$

where $\gamma_{M} \in M$ is defined by the limit

$$
\gamma_{M}=\lim _{\substack{M \subset M^{\prime} \in S_{L / K} \\\left[M^{\prime}: M\right] \rightarrow \infty}} N_{M^{\prime} / M}\left(\alpha_{M^{\prime}}+\beta_{M^{\prime}}\right),
$$

which exists in the local field $M$, for every $M \in S_{L / K}$.

Remark 4.2. Note that, for $\left(\alpha_{M}\right),\left(\beta_{M}\right) \in \mathbb{X}(L / K)$, the composition law

$$
\left(\left(\alpha_{M}\right),\left(\beta_{M}\right)\right) \mapsto\left(\alpha_{M}\right)+\left(\beta_{M}\right)=\left(\gamma_{M}\right)
$$

given by (4.1) is well defined, because $L / K$ is assumed to be an APF extension (cf. Theorem 2.1.3. in [17]).

This implies the following statement.

Theorem 4.3 (Fontaine-Wintenberger). Suppose $L / K$ is an APF extension. Then $\mathbb{X}(L / K)$ is a field under the addition

$$
+: \mathbb{X}(L / K) \times \mathbb{X}(L / K) \rightarrow \mathbb{X}(L / K)
$$

defined by (4.1) and under the multiplication

$$
\times: \mathbb{X}(L / K) \times \mathbb{X}(L / K) \rightarrow \mathbb{X}(L / K)
$$

defined naturally by the componentwise multiplication on $\mathbb{X}(L / K)^{\times}$. This field $\mathbb{X}(L / K)$ is called the field of norms corresponding to the APF extension $L / K$.

Now, in particular, we choose the following specific increasing directed family of subextensions $\left\{L_{i}\right\}_{0 \leq i \in \mathbb{Z}}$ in $L / K$ :

(i) $L_{0}$ is the maximal unramified extension of $K$ inside $L$;

(ii) $L_{1}$ is the maximal tamely ramified extension of $K$ inside $L$; 
(iii) for $i \geq 2$, the $L_{i}$ are chosen inductively as finite extensions of $L_{1}$ inside $L$ with $L_{i} \subseteq L_{i+1}$ and $\bigcup_{0 \leq i \in \mathbb{Z}} L_{i}=L$.

Observe that $L_{0} / K$ is a finite subextension of $L / K$ and, by the definition of tamely ramified extensions, $L_{0} \subseteq L_{1}$, with $\left[L_{1}: K\right]<\infty$. Thus, for any element $\left(\alpha_{L_{i}}\right)_{0 \leq i \in \mathbb{Z}}$ of $\mathbb{X}(L / K)$ we have

$$
\nu_{L_{i}}\left(\alpha_{L_{i}}\right)=\nu_{L_{0}}\left(\alpha_{L_{0}}\right)
$$

for every $0 \leq i \in \mathbb{Z}$. Therefore, the mapping

$$
\boldsymbol{\nu}_{\mathbb{X}(L / K)}: \mathbb{X}(L / K) \rightarrow \mathbb{Z} \cup\{\infty\}
$$

given by

$$
\boldsymbol{\nu}_{\mathbb{X}(L / K)}\left(\left(\alpha_{L_{i}}\right)_{0 \leq i \in \mathbb{Z}}\right)=\nu_{L_{0}}\left(\alpha_{L_{0}}\right)
$$

for $\left(\alpha_{L_{i}}\right)_{0 \leq i \in \mathbb{Z}} \in \mathbb{X}(L / K)$ is well defined; moreover, it is a discrete valuation on $\mathbb{X}(L / K)$, in view of (4.2).

Theorem 4.4 (Fontaine-Wintenberger). Suppose $L / K$ is an APF extension, and let $\mathbb{X}(L / K)$ be the field of norms attached to $L / K$. Then:

(i) the field $\mathbb{X}(L / K)$ is complete with respect to the discrete valuation $\boldsymbol{\nu}_{\mathbb{X}(L / K)}$ : $\mathbb{X}(L / K) \rightarrow \mathbb{Z} \cup\{\infty\}$ defined by (4.3);

(ii) the residue class field $\kappa_{\mathbb{X}(L / K)}$ of $\mathbb{X}(L / K)$ satisfies $\kappa_{\mathbb{X}(L / K)} \stackrel{\sim}{\longrightarrow} \kappa_{L}$;

(iii) the characteristic of the field $\mathbb{X}(L / K)$ is equal to char $\left(\kappa_{K}\right)$.

Proof. For a proof, look at Theorem 2.1.3 in [17.

Remark 4.5. As usual, the ring of integers $O_{\mathbb{X}(L / K)}$ of the local field (complete discrete valuation field) $\mathbb{X}(L / K)$ is defined by

$$
O_{\mathbb{X}(L / K)}=\left\{\left(\alpha_{L_{i}}\right)_{0 \leq i \in \mathbb{Z}} \in \mathbb{X}(L / K): \boldsymbol{\nu}_{\mathbb{X}(L / K)}\left(\left(\alpha_{L_{i}}\right)_{0 \leq i \in \mathbb{Z}}\right) \geq 0\right\} .
$$

Thus, by (4.3) and (4.2), for $\alpha=\left(\alpha_{L_{i}}\right)_{0 \leq i \in \mathbb{Z}} \in \mathbb{X}(L / K)$, the following two conditions are equivalent:

(i) $\left(\alpha_{L_{i}}\right)_{0 \leq i \in \mathbb{Z}} \in O_{\mathbb{X}(L / K)}$;

(ii) $\alpha_{L_{i}} \in O_{L_{i}}$ for every $0 \leq i \in \mathbb{Z}$.

The maximal ideal $\mathfrak{p}_{\mathbb{X}(L / K)}$ of $O_{\mathbb{X}(L / K)}$ is defined by

$$
\mathfrak{p}_{\mathbb{X}(L / K)}=\left\{\left(\alpha_{L_{i}}\right)_{0 \leq i \in \mathbb{Z}} \in \mathbb{X}(L / K): \boldsymbol{\nu}_{\mathbb{X}(L / K)}\left(\left(\alpha_{L_{i}}\right)_{0 \leq i \in \mathbb{Z}}\right) \geqslant 0\right\} .
$$

By (4.3) and (4.2), for $\alpha=\left(\alpha_{L_{i}}\right)_{0 \leq i \in \mathbb{Z}} \in \mathbb{X}(L / K)$, the following two conditions are equivalent:

(iii) $\left(\alpha_{L_{i}}\right)_{0 \leq i \in \mathbb{Z}} \in \mathfrak{p}_{\mathbb{X}(L / K)}$;

(iv) $\alpha_{L_{i}} \in \mathfrak{p}_{L_{i}}$ for every $0 \leq i \in \mathbb{Z}$.

The unit group $U_{\mathbb{X}(L / K)}$ of $O_{\mathbb{X}(L / K)}$ is defined by

$$
U_{\mathbb{X}(L / K)}=\left\{\left(\alpha_{L_{i}}\right)_{0 \leq i \in \mathbb{Z}} \in \mathbb{X}(L / K): \boldsymbol{\nu}_{\mathbb{X}(L / K)}\left(\left(\alpha_{L_{i}}\right)_{0 \leq i \in \mathbb{Z}}\right)=0\right\} .
$$

Again by (4.3) and (4.2), for $\alpha=\left(\alpha_{L_{i}}\right)_{0 \leq i \in \mathbb{Z}} \in \mathbb{X}(L / K)$, the following two conditions are equivalent:

(v) $\left(\alpha_{L_{i}}\right)_{0 \leq i \in \mathbb{Z}} \in U_{\mathbb{X}(L / K)}$;

(vi) $\alpha_{L_{i}} \in \bar{U}_{L_{i}}$ for every $0 \leq i \in \mathbb{Z}$.

Let $L / K$ be an infinite APF extension. Consider the tower

$$
K \subseteq F \subseteq L \subseteq E \subseteq K^{\text {sep }}
$$


of extensions over $K$, where $[F: K]<\infty$ and $[E: L]<\infty$. By parts (i) and (ii) of Lemma 3.3. it follows that $L / F$ is an infinite APF extension satisfying

$$
\mathbb{X}(L / K)=\mathbb{X}(L / F),
$$

by the definition of the field of norms, and $E / K$ is an infinite APF extension satisfying

$$
\mathbb{X}(L / K) \hookrightarrow \mathbb{X}(E / K)
$$

under the injective topological homomorphism

$$
\varepsilon_{L, E}^{(M)}: \mathbb{X}(L / K) \rightarrow \mathbb{X}(E / K),
$$

which depends on a finite extension $M$ over $K$ satisfying $L M=E$.

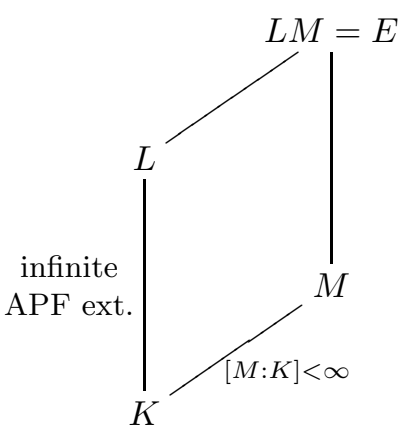

The topological embedding $\varepsilon_{L, E}^{(M)}: \mathbb{X}(L / K) \hookrightarrow \mathbb{X}(E / K)$ is defined as follows. Let $\left\{L_{i}\right\}_{0<i \in \mathbb{Z}}$ be an increasing directed family of subextensions in $L / K$ such that $\left[L_{i}: \bar{K}\right]<\infty$ for every $0 \leq i \in \mathbb{Z}$ and with $\bigcup_{0 \leq i \in \mathbb{Z}} L_{i}=L$. Then, clearly, $\left\{L_{i} M\right\}_{0 \leq i \in \mathbb{Z}}$ is an increasing directed family of subextensions in $E / K$ such that $\left[L_{i} M: K\right]<\infty$ for every $0 \leq i \in \mathbb{Z}$ and with $\bigcup_{0 \leq i \in \mathbb{Z}} L_{i} M=E$. For these two directed families, there exists a sufficiently large positive integer $m=m(M)$, which depends on the choice of $M$, such that

$$
N_{L_{j} M / L_{i} M}(x)=N_{L_{j} / L_{i}}(x)
$$

for $m \leq i \leq j$ and for each $x \in L_{j}$. Now, the topological embedding $\varepsilon_{L, E}^{(M)}: \mathbb{X}(L / K) \hookrightarrow$ $\mathbb{X}(E / K)$ is defined, for every $\left(\alpha_{L_{i}}\right)_{0 \leq i \in \mathbb{Z}} \in \mathbb{X}(L / K)-\{0\}$, by

$$
\varepsilon_{L, E}^{(M)}:\left(\alpha_{L_{i}}\right)_{0 \leq i \in \mathbb{Z}} \mapsto\left(\alpha_{L_{i} M}^{\prime}\right)_{0 \leq i \in \mathbb{Z}},
$$

where $\alpha_{L_{i} M}^{\prime} \in L_{i} M$ for every $0 \leq i \in \mathbb{Z}$,

$$
\alpha_{L_{i} M}^{\prime}= \begin{cases}\alpha_{L_{i}} & \text { if } i \geq m, \\ N_{L_{m} M / L_{i} M}\left(\alpha_{L_{m}}\right) & \text { if } \quad i<m .\end{cases}
$$

Thus, under the topological embedding

$$
\varepsilon_{L, E}^{(M)}: \mathbb{X}(L / K) \hookrightarrow \mathbb{X}(E / K)
$$

$\mathbb{X}(E / K) / \mathbb{X}(L / K)$ can be viewed as an extension of complete discrete valuation fields. At this point, the following remark is in order.

Remark 4.6. Let $L / K$ be an infinite APF extension and $E / L$ a finite extension. Suppose that $M$ and $M^{\prime}$ are two finite extensions over $K$ satisfying $L M=L M^{\prime}=E$. Then the embeddings $\varepsilon_{L, E}^{(M)}, \varepsilon_{L, E}^{\left(M^{\prime}\right)}: \mathbb{X}(L / K) \hookrightarrow \mathbb{X}(E / K)$ are the same. Therefore, as a notation, we set $\varepsilon_{L, E}^{(M)}=\varepsilon_{L, E}$. 
Now, given an infinite APF extension $L / K$, this time we let $E$ be a (not necessarily finite) separable extension of $L$. Let $S_{E / L}^{\mathrm{sep}}$ denote the partially ordered family of all finite separable subextensions in $E / L$. Then the following is true.

Proposition 4.7.

$$
\left\{\mathbb{X}\left(E^{\prime} / K\right) ; \varepsilon_{E^{\prime}, E^{\prime \prime}}: \mathbb{X}\left(E^{\prime} / K\right) \hookrightarrow \mathbb{X}\left(E^{\prime \prime} / K\right)\right\}_{E^{\prime}, E^{\prime \prime} \in S_{E / L}^{\mathrm{sep}}}
$$

is an inductive system under the topological embeddings

$$
\varepsilon_{E^{\prime}, E^{\prime \prime}}: \mathbb{X}\left(E^{\prime} / K\right) \hookrightarrow \mathbb{X}\left(E^{\prime \prime} / K\right)
$$

for $E^{\prime}, E^{\prime \prime} \in S_{E / L}^{\mathrm{sep}}$ with $E^{\prime} \subseteq E^{\prime \prime}$.

Let $\mathbb{X}(E, L / K)$ denote the topological field defined by the inductive limit

$$
\mathbb{X}(E, L / K)=\underset{E^{\prime} \in S_{E / L}^{\operatorname{sep}}}{\lim _{E / L}} \mathbb{X}\left(E^{\prime} / K\right)
$$

over the transition morphisms $\varepsilon_{E^{\prime}, E^{\prime \prime}}: \mathbb{X}\left(E^{\prime} / K\right) \hookrightarrow \mathbb{X}\left(E^{\prime \prime} / K\right)$ for $E^{\prime}, E^{\prime \prime} \in S_{E / L}^{\text {sep }}$ with $E^{\prime} \subseteq E^{\prime \prime}$.

The following theorem is central in the theory of fields of norms.

Theorem 4.8 (Fontaine-Wintenberger). Let $L / K$ be an $A P F$ extension and $E / L$ a Galois extension. Then $\mathbb{X}(E, L / K) / \mathbb{X}(L / K)$ is a Galois extension, and

$$
\operatorname{Gal}(\mathbb{X}(E, L / K) / \mathbb{X}(L / K)) \simeq \operatorname{Gal}(E / L)
$$

canonically.

An immediate and important consequence of this theorem is the following.

Corollary 4.9. Let $L / K$ be an APF extension. Then

$$
\operatorname{Gal}\left(\mathbb{X}\left(L^{\mathrm{sep}}, L / K\right) / \mathbb{X}(L / K)\right) \simeq \operatorname{Gal}\left(L^{\mathrm{sep}} / L\right)
$$

canonically.

\section{§5. Fesenko Reciprocity LAW}

In this section, we shall follow 1, 2, 3, to review the Fesenko reciprocity law for the local field $K$.

We recall the following definition (see [13]).

Definition 5.1. Let $\varphi=\varphi_{K} \in \operatorname{Gal}\left(K^{n r} / K\right)$ denote the Frobenius automorphism of $K$. An automorphism $\xi \in \operatorname{Gal}\left(K^{\text {sep }} / K\right)$ is called a Lubin-Tate splitting over $K$ if $\left.\xi\right|_{K^{n r}}=\varphi$.

Throughout the remainder of the text, we shall fix a Lubin-Tate splitting over the local field $K$, denoting it simply by $\varphi$, or by $\varphi_{K}$ if there is fear of confusion. Let $K_{\varphi}$ denote the fixed field $\left(K^{\text {sep }}\right)^{\varphi}$ of $\varphi \in G_{K}$ in $K^{\text {sep }}$.

Let $L / K$ be a totally ramified APF Galois extension satisfying

$$
K \subseteq L \subseteq K_{\varphi} .
$$

The field of norms $\mathbb{X}(L / K)$ is a local field by Theorem 4.4, Let $\widetilde{\mathbb{X}}(L / K)$ denote the completion $\widetilde{\mathbb{X}(L / K)}$ of $\mathbb{X}(L / K)^{n r}$ with respect to the valuation $\boldsymbol{\nu}_{\mathbb{X}(L / K)^{n r}}$, which is a unique extension of the valuation $\boldsymbol{\nu}_{\mathbb{X}(L / K)}$ to $\mathbb{X}(L / K)^{n r}$. As usual, we let $U_{\widetilde{\mathbb{X}}(L / K)}$ denote the unit group of the ring of integers $O_{\widetilde{\mathbb{X}}(L / K)}$ of the complete field $\widetilde{\mathbb{X}}(L / K)$. In this case, there exist isomorphisms

$$
\widetilde{\mathbb{X}}(L / K) \simeq \mathbb{F}_{p}^{\operatorname{sep}}((T))
$$


and

$$
U_{\widetilde{\mathbb{X}}(L / K)} \simeq \mathbb{F}_{p}^{\mathrm{sep}}[[T]]^{\times},
$$

defined by the machinery of Coleman power series (for the details, see Subsection 1.4 in [13]). Thus, the algebraic structures $\widetilde{\mathbb{X}}(L / K)$ and $U_{\widetilde{\mathbb{X}}(L / K)}$ initially seem to depend on the ground field $K$ only. However, as we shall state in Corollary 5.7, the law of composition on the "class formation", which is a certain subquotient of $U_{\widetilde{\mathbb{X}}(L / K)}$, does indeed depend on the $\operatorname{Gal}(L / K)$-module structure of $U_{\widetilde{\mathbb{X}}(L / K)}$.

Remark 5.2. The problem of eliminating this dependence on the Galois-module structure of $U_{\widetilde{\mathbb{X}}(L / K)}$ is closely related to Sen's infinite-dimensional Hodge-Tate theory [11], or more generally, with the $p$-adic Langlands program.

As in $\S 1$, let $\widetilde{K}$ denote the completion of $K^{n r}$ with respect to the valuation $\nu_{K^{n r}}$ on $K^{n r}$, and let $\widetilde{L}=L \widetilde{K}$. Then $\widetilde{L} / \widetilde{K}$ is an APF extension, because $L / K$ is an APF extension, and the corresponding field of norms satisfies

$$
\mathbb{X}(\widetilde{L} / \widetilde{K})=\widetilde{\mathbb{X}}(L / K) .
$$

Now, let

$$
\operatorname{Pr}_{\widetilde{K}}: U_{\widetilde{\mathbb{X}}(L / K)} \rightarrow U_{\widetilde{K}}
$$

denote the projection map to the $\widetilde{K}$-coordinate of $U_{\widetilde{\mathbb{X}}(L / K)}$ under the identification described in (5.2). Throughout the text, $U_{\widetilde{\mathbb{X}}(L / K)}^{1}$ stands for the kernel $\operatorname{ker}\left(\operatorname{Pr}_{\widetilde{K}}\right)$ of the projection map $\operatorname{Pr}_{\widetilde{K}}: U_{\widetilde{\mathbb{X}}(L / K)} \rightarrow U_{\widetilde{K}}$.

Definition 5.3. The subgroup

$$
\operatorname{Pr}_{\widetilde{K}}^{-1}\left(U_{K}\right)=\left\{U \in U_{\widetilde{\mathbb{X}}(L / K)}: \operatorname{Pr}_{\widetilde{K}}(U) \in U_{K}\right\}
$$

of $U_{\widetilde{\mathbb{X}}(L / K)}$ is called the Fesenko diamond subgroup of $U_{\widetilde{\mathbb{X}}(L / K)}$ and is denoted by $U_{\widetilde{\mathbb{X}}(L / K)}$.

Now, as in [1, 2, 3], we choose an ascending chain of field extensions

$$
K=E_{o} \subset E_{1} \subset \cdots \subset E_{i} \subset \cdots \subset L
$$

in such a way that

(i) $L=\bigcup_{0 \leq i \in \mathbb{Z}} E_{i}$;

(ii) $E_{i} / K$ is a Galois extension for each $0 \leq i \in \mathbb{Z}$;

(iii) $E_{i+1} / E_{i}$ is cyclic of prime degree $\left[E_{i+1}: E_{i}\right]=p=\operatorname{char}\left(\kappa_{K}\right)$ for each $1 \leq i \in \mathbb{Z}$;

(iv) $E_{1} / E_{o}$ is cyclic of degree relatively prime to $p$.

Such a sequence $\left(E_{i}\right)_{0 \leq i \in \mathbb{Z}}$ exists (because $L / K$ is a solvable Galois extension) and will be called a basic ascending chain of subextensions in $L / K$. Then, we can construct $\mathbb{X}(L / K)$ by the basic sequence $\left(E_{i}\right)_{0 \leq i \in \mathbb{Z}}$ and $\widetilde{\mathbb{X}}(L / K)$ by $\left(\widetilde{E}_{i}\right)_{0 \leq i \in \mathbb{Z}}$. Note that the Galois group $\operatorname{Gal}(L / K)$ corresponding to the extension $L / K$ acts continuously on $\mathbb{X}(L / K)$ and on $\widetilde{\mathbb{X}}(L / K)$ naturally, if we define the Galois action of $\sigma \in \operatorname{Gal}(L / K)$ on the chain

$$
K=E_{o} \subset E_{1} \subset \cdots \subset E_{i} \subset \cdots \subset L
$$

by the action of $\sigma$ on each $E_{i}$ for $0 \leq i \in \mathbb{Z}$ as

$$
K=E_{o}^{\sigma} \subset E_{1}^{\sigma}=E_{1} \subset \cdots \subset E_{i}^{\sigma}=E_{i} \subset \cdots \subset L,
$$

and respectively on the chain

$$
\widetilde{K}=\widetilde{K} E_{o} \subset \widetilde{E}_{1}=\widetilde{K} E_{1} \subset \cdots \subset \widetilde{E}_{i}=\widetilde{K} E_{i} \subset \cdots \subset \widetilde{L}=\widetilde{K} L
$$


by the action of $\sigma$ on the " $E_{i}$-part" of each $\widetilde{E}_{i}$ (note that $E_{i} \cap K^{n r}=K$ )

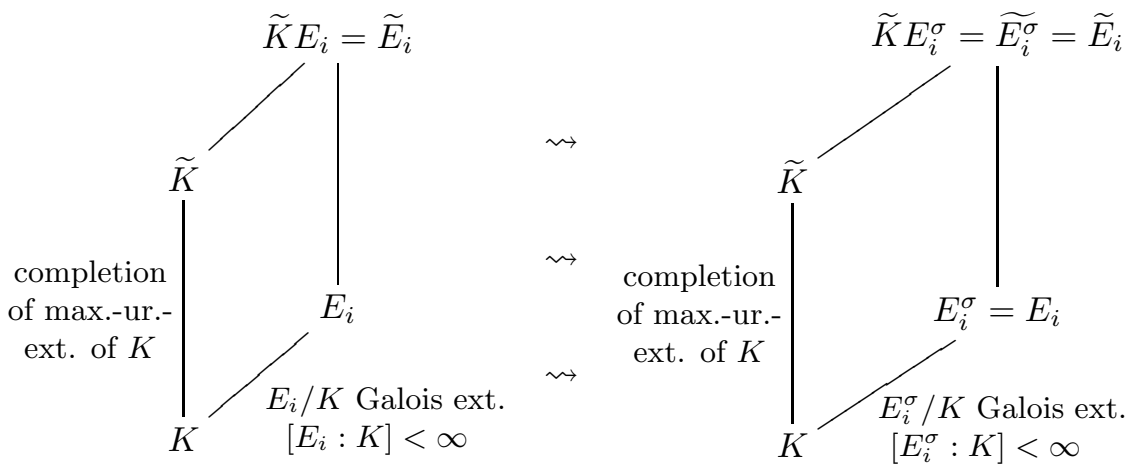

for $0 \leq i \in \mathbb{Z}$ as

$$
\widetilde{K}=\widetilde{K} E_{o}^{\sigma} \subset \widetilde{K} E_{1}^{\sigma}=\widetilde{K} E_{1} \subset \cdots \subset \widetilde{K} E_{i}^{\sigma}=\widetilde{K} E_{i} \subset \cdots \subset \widetilde{K} L .
$$

Therefore, there exist natural continuous actions of $\operatorname{Gal}(L / K)$ on $U_{\mathbb{X}(L / K)}$, on $U_{\widetilde{\mathbb{X}}(L / K)}$, and on $U_{\widetilde{\mathbb{X}}(L / K)}^{\diamond}$ compatible with the respective topological group structures, so that we shall always view them as topological $\mathrm{Gal}(L / K)$-modules in this text. Now, we recall the following theorem about norm compatible sequences of prime elements (cf. [13).

Theorem 5.4 (Koch-de Shalit). Assume that $K \subseteq L \subset K_{\varphi}$. Then for any chain

$$
K=E_{o} \subset E_{1} \subset \cdots \subset E_{i} \subset \cdots \subset L
$$

of finite subextensions of $L / K$, there exists a unique norm-compatible sequence

$$
\pi_{E_{o}}, \pi_{E_{1}}, \ldots, \pi_{E_{i}}, \ldots
$$

where each $\pi_{E_{i}}$ is a prime element of $E_{i}$ for $0 \leq i \in \mathbb{Z}$.

In view of the theorem of Koch and de Shalit, we introduce the natural prime element $\Pi_{\varphi ; L / K}$ of the local field $\mathbb{X}(L / K)$ (which depends on the fixed Lubin-Tate splitting $\varphi$ (cf. [13]) as well as on the subextension $L / K$ of $K_{\varphi} / K$ ) by

$$
\Pi_{\varphi ; L / K}=\left(\pi_{E_{i}}\right)_{0 \leq i \in \mathbb{Z}} .
$$

By the theorem of Koch and de Shalit, the prime element $\Pi_{\varphi ; L / K}$ of $\mathbb{X}(L / K)$ does not depend on the choice of a chain $\left(E_{i}\right)_{0 \leq i \in \mathbb{Z}}$ of finite subextensions of $L / K$.

Theorem 5.5 (Fesenko). For each $\sigma \in \operatorname{Gal}(L / K)$, there exists $U_{\sigma} \in U_{\widetilde{\mathbb{X}}(L / K)}^{\diamond}$ that solves the equation

$$
U^{1-\varphi}=\Pi_{\varphi ; L / K}^{\sigma-1}
$$

for $U$. Moreover, the solution set of this equation consists of elements in the coset $U_{\sigma} . U_{\mathbb{X}(L / K)}$ of $U_{\sigma}$ modulo $U_{\mathbb{X}(L / K)}$.

In fact, for the most general form of this theorem and its proof, see 9 .

Now, define the arrow

$$
\phi_{L / K}^{(\varphi)}: \operatorname{Gal}(L / K) \rightarrow U_{\widetilde{\mathbb{X}}(L / K)}^{\diamond} / U_{\mathbb{X}(L / K)}
$$

by

$$
\phi_{L / K}^{(\varphi)}: \sigma \mapsto \bar{U}_{\sigma}=U_{\sigma} . U_{\mathbb{X}(L / K)},
$$

for every $\sigma \in \operatorname{Gal}(L / K)$. 
Theorem 5.6 (Fesenko). The arrow

$$
\phi_{L / K}^{(\varphi)}: \operatorname{Gal}(L / K) \rightarrow U_{\widetilde{\mathbb{X}}(L / K)}^{\diamond} / U_{\mathbb{X}(L / K)}
$$

defined for the extension $L / K$ is injective, and for every $\sigma, \tau \in \operatorname{Gal}(L / K)$ we have

$$
\phi_{L / K}^{(\varphi)}(\sigma \tau)=\phi_{L / K}^{(\varphi)}(\sigma) \phi_{L / K}^{(\varphi)}(\tau)^{\sigma},
$$

i.e., the cocycle condition is satisfied.

We formulate a natural consequence of this theorem, denoting the image set of the mapping $\phi_{L / K}^{(\varphi)}$ by $\operatorname{im}\left(\phi_{L / K}^{(\varphi)}\right) \subseteq U_{\widetilde{\mathbb{X}}(L / K)}^{\diamond} / U_{\mathbb{X}(L / K)}$.

Corollary 5.7. Define a law of composition $*$ on $\operatorname{im}\left(\phi_{L / K}^{(\varphi)}\right)$ by

$$
\bar{U} * \bar{V}=\bar{U} \cdot \bar{V}^{\left(\phi_{L / K}^{(\varphi)}\right)^{-1}(\bar{U})}
$$

for every $\bar{U}, \bar{V} \in \operatorname{im}\left(\phi_{L / K}^{(\varphi)}\right)$. Then $\operatorname{im}\left(\phi_{L / K}^{(\varphi)}\right)$ is a topological group under $*$, and the map $\phi_{L / K}^{(\varphi)}$ induces an isomorphism of topological groups

$$
\phi_{L / K}^{(\varphi)}: \operatorname{Gal}(L / K) \stackrel{\sim}{\longrightarrow} \operatorname{im}\left(\phi_{L / K}^{(\varphi)}\right),
$$

where the topological group structure on $\operatorname{im}\left(\phi_{L / K}^{(\varphi)}\right)$ is defined with respect to the binary operation $*$ described by (5.12).

Now, for each $0 \leq i \in \mathbb{R}$, consider the $i$ th higher unit group $U_{\widetilde{\mathbb{X}}(L / K)}^{i}$ of the field $\widetilde{\mathbb{X}}(L / K)$, and define the group

$$
\left(U_{\widetilde{\mathbb{X}}(L / K)}^{\diamond}\right)^{i}=U_{\widetilde{\mathbb{X}}(L / K)}^{\diamond} \cap U_{\widetilde{\mathbb{X}}(L / K)}^{i} .
$$

Theorem 5.8 (Fesenko ramification theorem). For $0 \leq n \in \mathbb{Z}$, let $\operatorname{Gal}(L / K)_{n}$ denote the nth higher ramification subgroup of the Galois group $\operatorname{Gal}(L / K)$ corresponding to the $A P F$ Galois subextension $L / K$ of $K_{\varphi} / K$ in the lower numbering. Then, we have the inclusion

$$
\begin{aligned}
\phi_{L / K}^{(\varphi)} & \left(\operatorname{Gal}(L / K)_{n}-\operatorname{Gal}(L / K)_{n+1}\right) \\
& \subseteq\left(U_{\widetilde{\mathbb{X}}(L / K)}^{\diamond}\right)^{n} U_{\mathbb{X}(L / K)} / U_{\mathbb{X}(L / K)}-\left(U_{\widetilde{\mathbb{X}}(L / K)}^{\diamond}\right)^{n+1} U_{\mathbb{X}(L / K)} / U_{\mathbb{X}(L / K)} .
\end{aligned}
$$

Now, let $M / K$ be a Galois subextension of $L / K$. Thus, there exists a chain of field extensions

$$
K \subseteq M \subseteq L \subseteq K_{\varphi},
$$

where $M$ is a totally ramified APF Galois extension over $K$ by Lemma 3.3, Let

$$
\phi_{M / K}^{(\varphi)}: \operatorname{Gal}(M / K) \rightarrow U_{\widetilde{\mathbb{X}}(M / K)}^{\diamond} / U_{\mathbb{X}(M / K)}
$$

be the corresponding map defined for the extension $M / K$.

Now, let

$$
K=E_{o} \subset E_{1} \subset \cdots \subset E_{i} \subset \cdots \subset L
$$

be an ascending chain satisfying $L=\bigcup_{0 \leq i \in \mathbb{Z}} E_{i}$ and $\left[E_{i+1}: E_{i}\right]<\infty$ for every $0 \leq i \in \mathbb{Z}$. Then

$$
K=E_{o} \cap M \subseteq E_{1} \cap M \subseteq \cdots \subseteq E_{i} \cap M \subseteq \cdots \subset M
$$

is an ascending chain of field extensions satisfying $M=\bigcup_{0 \leq i \in \mathbb{Z}}\left(E_{i} \cap M\right)$ and $\left[E_{i+1} \cap M\right.$ : $\left.E_{i} \cap M\right]<\infty$ for every $0 \leq i \in \mathbb{Z}$. Thus, we can construct $\mathbb{X}(M / K)$ by the sequence 
$\left(E_{i} \cap M\right)_{0 \leq i \in \mathbb{Z}}$ and $\widetilde{\mathbb{X}}(M / K)$ by the sequence $\left(\widetilde{E_{i} \cap M}\right)_{0 \leq i \in \mathbb{Z}}$. Furthermore, for every pair $0 \leq i, i^{\prime} \in \mathbb{Z}$ satisfying $i \leq i^{\prime}$, the commutative square

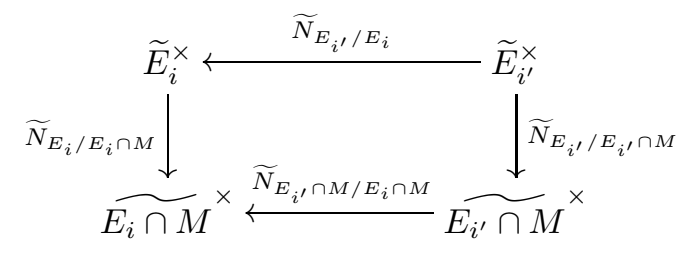

induces a group homomorphism

$$
\widetilde{\mathcal{N}}_{L / M}=\lim _{0 \leq i \in \mathbb{Z}} \tilde{N}_{E_{i} / E_{i} \cap M}: \widetilde{\mathbb{X}}(L / K)^{\times} \rightarrow \widetilde{\mathbb{X}}(M / K)^{\times}
$$

defined by

$$
\widetilde{\mathcal{N}}_{L / M}\left(\left(\alpha_{\widetilde{E}_{i}}\right)_{0 \leq i \in \mathbb{Z}}\right)=\left(\widetilde{N}_{E_{i} / E_{i} \cap M}\left(\alpha_{\widetilde{E}_{i}}\right)\right)_{0 \leq i \in \mathbb{Z}},
$$

for every $\left(\alpha_{\widetilde{E}_{i}}\right)_{0 \leq i \in \mathbb{Z}} \in \widetilde{\mathbb{X}}(L / K)^{\times}$.

Remark 5.9. The group homomorphism

$$
\widetilde{\mathcal{N}}_{L / M}: \widetilde{\mathbb{X}}(L / K)^{\times} \rightarrow \widetilde{\mathbb{X}}(M / K)^{\times}
$$

defined by (5.15) and (5.16) does not depend on the choice of an ascending chain

$$
K=E_{o} \subset E_{1} \subset \cdots \subset E_{i} \subset \cdots \subset L
$$

satisfying $L=\bigcup_{0 \leq i \in \mathbb{Z}} E_{i}$ and $\left[E_{i+1}: E_{i}\right]<\infty$ for every $0 \leq i \in \mathbb{Z}$.

The basic properties of this group homomorphism are the following.

(i) If $U=\left(u_{\widetilde{E}_{i}}\right)_{0 \leq i \in \mathbb{Z}} \in U_{\widetilde{\mathbb{X}}(L / K)}$, then $\widetilde{\mathcal{N}}_{L / M}(U) \in U_{\widetilde{\mathbb{X}}(M / K)}$.

Proof. The definition of the valuation $\nu_{\widetilde{\mathbb{X}}(M / K)}$ of $\widetilde{\mathbb{X}}(M / K)$ and the definition of the valuation $\nu_{\widetilde{\mathbb{X}}(L / K)}$ of $\widetilde{\mathbb{X}}(L / K)$ show that

$$
\nu_{\widetilde{\mathbb{X}}(M / K)}\left(\widetilde{\mathcal{N}}_{L / M}(U)\right)=\nu_{\widetilde{\mathbb{X}}(M / K)}\left(\left(\widetilde{N}_{E_{i} / E_{i} \cap M}\left(u_{\widetilde{E}_{i}}\right)\right)_{0 \leq i \in \mathbb{Z}}\right)=\nu_{\widetilde{K}}\left(u_{\widetilde{K}}\right)=0,
$$

as

$$
\nu_{\widetilde{\mathbb{X}}(L / K)}(U)=\nu_{\widetilde{K}}\left(u_{\widetilde{K}}\right)=0
$$

since $U \in U_{\widetilde{\mathbb{X}}(L / K)}$.

(ii) If $U=\left(u_{\widetilde{E}_{i}}\right)_{0 \leq i \in \mathbb{Z}} \in U_{\widetilde{\mathbb{X}}(L / K)}^{\diamond}$, then $\widetilde{\mathcal{N}}_{L / M}(U) \in U_{\widetilde{\mathbb{X}}(M / K)}^{\diamond}$.

Proof. The assertion follows by observing that $\operatorname{Pr}_{\widetilde{K}}(U)=u_{\widetilde{K}}$ and $\operatorname{Pr}_{\widetilde{K}}\left(\widetilde{\mathcal{N}}_{L / M}(U)\right)=$ $\widetilde{N}_{E_{o} / E_{o} \cap M}\left(u_{\widetilde{E}_{o}}\right)=u_{\widetilde{K}} \in U_{K}$.

(iii) If $U=\left(u_{E_{i}}\right)_{0 \leq i \in \mathbb{Z}} \in U_{\mathbb{X}(L / K)}$, then $\widetilde{\mathcal{N}}_{L / M}(U) \in U_{\mathbb{X}(M / K)}$.

Proof. The assertion follows by the definition (5.16) of the homomorphism (5.15), combined with the fact that $\widetilde{N}_{E_{i} / E_{i} \cap M}\left(u_{E_{i}}\right)=N_{E_{i} / E_{i} \cap M}\left(u_{E_{i}}\right)$ for every $u_{E_{i}} \in U_{E_{i}}$ and every $0 \leq i \in \mathbb{Z}$. 
Thus, the group homomorphism (5.15) defined by (5.16) induces a group homomorphism, which will be called the Coleman norm map from $L$ to $M$,

$$
\tilde{\mathcal{N}}_{L / M}^{\text {Coleman }}: U_{\widetilde{\mathbb{X}}(L / K)}^{\diamond} / U_{\mathbb{X}(L / K)} \rightarrow U_{\widetilde{\mathbb{X}}(M / K)}^{\diamond} / U_{\mathbb{X}(M / K)},
$$

and is defined by

$$
\tilde{\mathcal{N}}_{L / M}^{\text {Coleman }}(\bar{U})=\tilde{\mathcal{N}}_{L / M}(U) \cdot U_{\mathbb{X}(M / K)}
$$

for every $U \in U_{\widetilde{\mathbb{X}}(L / K)}^{\diamond}$; as before, $\bar{U}$ denotes the coset $U \cdot U_{\mathbb{X}(L / K)}$ in $U_{\widetilde{\mathbb{X}}(L / K)}^{\diamond} / U_{\mathbb{X}(L / K)}$.

The following theorem was stated in Fesenko's papers [1, 2, 3, without proof. Thus, for completeness, we shall supply a proof of this theorem as well.

Theorem 5.10 (Fesenko). For the Galois subextension $M / K$ of $L / K$, the square

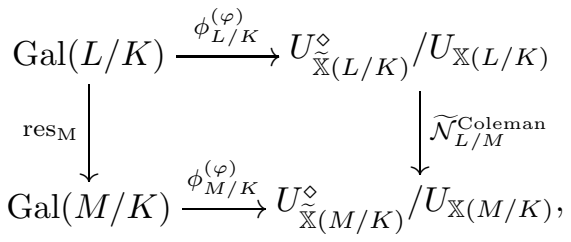

where the right vertical arrow

$$
\widetilde{\mathcal{N}}_{L / M}^{\text {Coleman }}: U_{\widetilde{\mathbb{X}}(L / K)}^{\diamond} / U_{\mathbb{X}(L / K)} \rightarrow U_{\widetilde{\mathbb{X}}(M / K)}^{\diamond} / U_{\mathbb{X}(M / K)}
$$

is the Coleman norm map from $L$ to $M$ defined by (5.17) and (5.18), is commutative.

Proof. For each $\sigma \in \operatorname{Gal}(L / K)$, we must show that

$$
\tilde{\mathcal{N}}_{L / M}^{\text {Coleman }}\left(\phi_{L / K}^{(\varphi)}(\sigma)\right)=\phi_{M / K}^{(\varphi)}\left(\left.\sigma\right|_{M}\right)
$$

Thus, it suffices to prove the congruence

$$
\widetilde{\mathcal{N}}_{L / M}\left(U_{\sigma}\right) \equiv U_{\left.\sigma\right|_{M}} \quad\left(\bmod U_{\mathbb{X}(M / K)}\right),
$$

or equivalently, it suffices to prove that

$$
\frac{\tilde{\mathcal{N}}_{L / M}\left(U_{\sigma}\right)}{\widetilde{\mathcal{N}}_{L / M}\left(U_{\sigma}\right)^{\varphi}}=\frac{\Pi_{\varphi ; M / K}^{\left.\sigma\right|_{M}}}{\Pi_{\varphi ; M / K}}
$$

Now, without loss of generality, by Remark 5.9, the ascending chain of extensions

$$
K=E_{o} \subset E_{1} \subset \cdots \subset E_{i} \subset \cdots \subset L
$$

can be chosen as the basic sequence introduced at the beginning of this section. Thus, each extension $E_{i} / K$ is finite and Galois for $0 \leq i \in \mathbb{Z}$. Now, let $U_{\sigma}=\left(u_{\widetilde{E}_{i}}\right)_{0 \leq i \in \mathbb{Z}} \in$ $U_{\widetilde{\mathbb{X}}(L / K)}^{\diamond}$. Then, for each $0 \leq i \in \mathbb{Z}$,

$$
\frac{\widetilde{N}_{E_{i} / E_{i} \cap M}\left(u_{\widetilde{E}_{i}}\right)}{\widetilde{N}_{E_{i} / E_{i} \cap M}\left(u_{\widetilde{E}_{i}}\right)^{\varphi}}=\frac{\widetilde{N}_{E_{i} / E_{i} \cap M}\left(u_{\widetilde{E}_{i}}\right)}{\widetilde{N}_{E_{i} / E_{i} \cap M}\left(u_{\widetilde{E}_{i}}^{\varphi}\right)}=\widetilde{N}_{E_{i} / E_{i} \cap M}\left(\frac{u_{\widetilde{E}_{i}}}{u_{\widetilde{E}_{i}}^{\varphi}}\right) .
$$

Next, the relation $\frac{u_{\widetilde{E}_{i}}}{u_{\mathbb{E}_{i}}^{\varphi}}=\frac{\pi_{E_{i}}^{\sigma}}{\pi_{E_{i}}}$, which follows from $\frac{U_{\sigma}}{U_{\sigma}^{\varphi}}=\frac{\Pi_{\varphi ; L / K}^{\sigma}}{\Pi_{\varphi ; L / K}}$, yields

$$
\frac{\widetilde{N}_{E_{i} / E_{i} \cap M}\left(u_{\widetilde{E}_{i}}\right)}{\widetilde{N}_{E_{i} / E_{i} \cap M}\left(u_{\widetilde{E}_{i}}\right)^{\varphi}}=\widetilde{N}_{E_{i} / E_{i} \cap M}\left(\frac{\pi_{E_{i}}^{\sigma}}{\pi_{E_{i}}}\right) .
$$


Thus, by the theorem of Koch and de Shalit, it follows that

$$
\widetilde{N}_{E_{i} / E_{i} \cap M}\left(\frac{\pi_{E_{i}}^{\sigma}}{\pi_{E_{i}}}\right)=\frac{\widetilde{N}_{E_{i} / E_{i} \cap M}\left(\pi_{E_{i}}\right)^{\sigma}}{\widetilde{N}_{E_{i} / E_{i} \cap M}\left(\pi_{E_{i}}\right)}=\frac{\pi_{E_{i} \cap M}^{\left.\sigma\right|_{M}}}{\pi_{E_{i} \cap M}},
$$

which proves the formula

$$
\frac{\widetilde{\mathcal{N}}_{L / M}\left(U_{\sigma}\right)}{\widetilde{\mathcal{N}}_{L / M}\left(U_{\sigma}\right)^{\varphi}}=\frac{\Pi_{\varphi ; M / K}^{\left.\sigma\right|_{M}}}{\Pi_{\varphi ; M / K}} .
$$

The proof is complete.

Now, let $F / K$ be a finite subextension of $L / K$. Then, since $F$ is compatible with $(K, \varphi)$ in the sense of [13, p. 89], we may fix the Lubin-Tate splitting over $F$ to be $\varphi_{F}=\varphi_{K}=\varphi$. Thus, there exists a chain of field extensions

$$
K \subseteq F \subseteq L \subseteq K_{\varphi} \subseteq F_{\varphi}
$$

where $L$ is a totally ramified APF Galois extension over $F$ by Lemma 3.3 , So, there exists a mapping

$$
\phi_{L / F}^{(\varphi)}: \operatorname{Gal}(L / F) \rightarrow U_{\widetilde{\mathbb{X}}(L / F)}^{\diamond} / U_{\mathbb{X}(L / F)}
$$

corresponding to the extension $L / F$.

For the APF extension $L / F$, we fix an ascending chain

$$
F=F_{o} \subset F_{1} \subset \cdots \subset F_{i} \subset \cdots \subset L
$$

satisfying $L=\bigcup_{0 \leq i \in \mathbb{Z}} F_{i}$ and $\left[F_{i+1}: F_{i}\right]<\infty$ for every $0 \leq i \in \mathbb{Z}$. We introduce a homomorphism

$$
\Lambda_{F / K}: \widetilde{\mathbb{X}}(L / F)^{\times} \rightarrow \widetilde{\mathbb{X}}(L / K)^{\times}
$$

by

$$
\begin{aligned}
\Lambda_{F / K} & :\left(\alpha_{F} \stackrel{\widetilde{N}_{F_{1} / F}}{\longleftarrow} \alpha_{F_{1}} \stackrel{\widetilde{N}_{F_{2} / F_{1}}}{\longleftarrow} \cdots\right) \\
& \mapsto\left(\widetilde{N}_{F / K}\left(\alpha_{F}\right) \stackrel{\widetilde{N}_{F / K}}{\longleftarrow} \alpha_{F} \stackrel{\widetilde{N}_{F_{1} / F}}{\longleftarrow} \alpha_{F_{1}} \stackrel{\widetilde{N}_{F_{2} / F_{1}}}{\longleftarrow} \cdots\right)
\end{aligned}
$$

for each $\left(\alpha_{F_{i}}\right)_{0 \leq i \in \mathbb{Z}} \in \widetilde{\mathbb{X}}(L / F)^{\times}$.

Remark 5.11. It is clear that the homomorphism

$$
\Lambda_{F / K}: \widetilde{\mathbb{X}}(L / F)^{\times} \rightarrow \widetilde{\mathbb{X}}(L / K)^{\times}
$$

defined by (5.20) and (5.21) does not depend on the choice of an ascending chain of fields

$$
F=F_{o} \subset F_{1} \subset \cdots \subset F_{i} \subset \cdots \subset L
$$

satisfying $L=\bigcup_{0 \leq i \in \mathbb{Z}} F_{i}$ and $\left[F_{i+1}: F_{i}\right]<\infty$ for every $0 \leq i \in \mathbb{Z}$.

The basic properties of this group homomorphism are as follows.

(i) The square

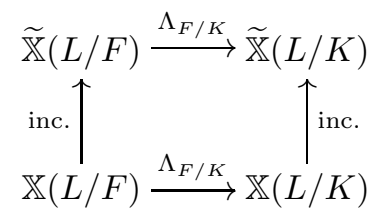

is commutative.

(ii) If $U=\left(u_{\widetilde{F}_{i}}\right)_{0 \leq i \in \mathbb{Z}} \in U_{\widetilde{\mathbb{X}}(L / F)}$, then $\Lambda_{F / K}(U) \in U_{\widetilde{\mathbb{X}}(L / K)}$. 
(iii) If $U=\left(u_{\widetilde{F}_{i}}\right)_{0 \leq i \in \mathbb{Z}} \in U_{\widetilde{\mathbb{X}}(L / F)}^{\diamond}$, then $\Lambda_{F / K}(U) \in U_{\widetilde{\mathbb{X}}(L / K)}^{\diamond}$.

(iv) If $U=\left(u_{F_{i}}\right)_{0 \leq i \in \mathbb{Z}} \in U_{\mathbb{X}(L / F)}$, then $\Lambda_{F / K}(U) \in U_{\mathbb{X}(L / K)}$.

Thus, the group homomorphism (5.20) defined by (5.21) induces the group homomorphism

$$
\lambda_{F / K}: U_{\widetilde{\mathbb{X}}(L / F)}^{\diamond} / U_{\mathbb{X}(L / F)} \rightarrow U_{\widetilde{\mathbb{X}}(L / K)}^{\diamond} / U_{\mathbb{X}(L / K)}
$$

defined by

$$
\lambda_{F / K}: \bar{U} \mapsto \Lambda_{F / K}(U) \cdot U_{\mathbb{X}(L / K)},
$$

for every $U \in U_{\widetilde{\mathbb{X}}(L / F)}^{\diamond}$; as before, $\bar{U}$ denotes the coset $U \cdot U_{\mathbb{X}(L / F)}$ in $U_{\widetilde{\mathbb{X}}(L / F)}^{\diamond} / U_{\mathbb{X}(L / F)}$.

The following theorem was stated in [1, 2, 3] without proof. Thus, for completeness, we shall supply a proof of this theorem as well.

Theorem 5.12 (Fesenko). For the finite subextension $F / K$ of $L / K$, the square

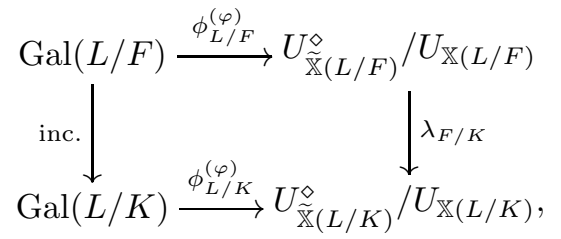

where the right vertical arrow $\lambda_{F / K}: U_{\widetilde{\mathbb{X}}(L / F)}^{\diamond} / U_{\mathbb{X}(L / F)} \rightarrow U_{\widetilde{\mathbb{X}}(L / K)}^{\diamond} / U_{\mathbb{X}(L / K)}$ is defined by (5.22) and (5.23), is commutative.

Proof. For $\sigma \in \operatorname{Gal}(L / F)$, we have $\phi_{L / F}^{(\varphi)}(\sigma)=U_{\sigma} \cdot U_{\mathbb{X}(L / F)}$, where $U_{\sigma} \in U_{\widetilde{\mathbb{X}}(L / F)}^{\diamond}$ satisfies

$$
\frac{U_{\sigma}}{U_{\sigma}^{\varphi}}=\frac{\Pi_{\varphi ; L / F}^{\sigma}}{\Pi_{\varphi ; L / F}} .
$$

Here, $\Pi_{\varphi ; L / F}$ is the norm compatible sequence of primes $\left(\pi_{F_{i}}\right)_{0 \leq i \in \mathbb{Z}}$. Now,

$$
\Lambda_{F / K}\left(\frac{U_{\sigma}}{U_{\sigma}^{\varphi}}\right)=\frac{\Lambda_{F / K}\left(U_{\sigma}\right)}{\Lambda_{F / K}\left(U_{\sigma}^{\varphi}\right)}=\frac{\Lambda_{F / K}\left(U_{\sigma}\right)}{\Lambda_{F / K}\left(U_{\sigma}\right)^{\varphi}} .
$$

On the other hand, $\Lambda_{F / K}\left(\Pi_{\varphi ; L / F}\right)=\Pi_{\varphi ; L / K}$ and $\Lambda_{F / K}\left(\Pi_{\varphi ; L / F}^{\sigma}\right)=\Pi_{\varphi ; L / K}^{\sigma}$. Thus, (5.25) yields

which shows that

$$
\frac{\Lambda_{F / K}\left(U_{\sigma}\right)}{\Lambda_{F / K}\left(U_{\sigma}\right)^{\varphi}}=\frac{\Pi_{\varphi ; L / K}^{\sigma}}{\Pi_{\varphi ; L / K}}
$$

$$
\phi_{L / K}^{(\varphi)}(\sigma)=\Lambda_{F / K}\left(U_{\sigma}\right) \cdot U_{\mathbb{X}(L / K)}=\lambda_{F / K}\left(\phi_{L / F}^{(\varphi)}(\sigma)\right),
$$

completing the proof of the commutativity of the square.

Furthermore, if $L / K$ is a finite extension, then the composition

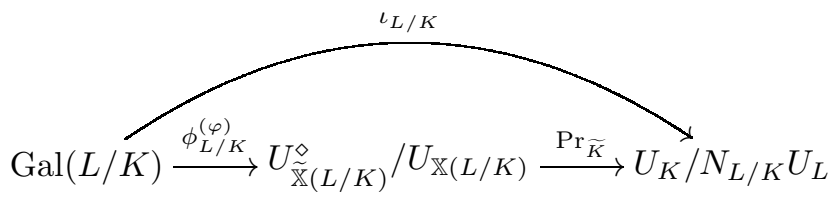

is the Iwasawa-Neukirch map of the extension $L / K$. Thus, the mapping $\phi_{L / K}^{(\varphi)}$ defined for $L / K$ is a generalization of the Iwasawa-Neukirch map $\iota_{L / K}: \operatorname{Gal}(L / K) \rightarrow$ $U_{K} / N_{L / K}\left(U_{L}\right)$ for the totally ramified APF Galois subextensions $L / K$ of $K_{\varphi} / K$. 
Likewise, the definition of the Hazewinkel map $h_{L / K}: U_{K} / N_{L / K} U_{L} \rightarrow \operatorname{Gal}(L / K)^{a b}$ (formulated initially for totally ramified finite Galois extensions $L / K$ ) can be extended to the totally ramified APF Galois subextensions of $K_{\varphi} / K$ by generalizing the Serre short exact sequence introduced in (1.1) and (1.2). In order to do so, first we need to assume that the local field $K$ satisfies the condition

$$
\boldsymbol{\mu}_{p}\left(K^{\mathrm{sep}}\right)=\left\{\alpha \in K^{\mathrm{sep}}: \alpha^{p}=1\right\} \subset K,
$$

where $p=\operatorname{char}\left(\kappa_{K}\right)$.

Remark 5.13. If $K$ is a local field of characteristic $p=\operatorname{char}\left(\kappa_{K}\right)$, the assumption (5.26) on $K$ is satisfied automatically. For the details on the assumption (5.26) on $K$, we refer the reader to [1, 2, 3].

In what follows, as before, we let $L / K$ be a totally ramified APF Galois extension satisfying (5.1). Under this assumption, there exists a topological $\mathrm{Gal}(L / K)$-submodule $Y_{L / K}$ of $U_{\widetilde{\mathbb{X}}(L / K)}^{\diamond}$ such that

(i) $U_{\mathbb{X}(L / K)} \subseteq Y_{L / K}$;

(ii) the composition

$$
\Phi_{L / K}^{(\varphi)}: \operatorname{Gal}(L / K) \stackrel{\phi_{L / K}^{(\varphi)}}{\longrightarrow} U_{\widetilde{\mathbb{X}}(L / K)}^{\diamond} / U_{\mathbb{X}(L / K)} \underset{\begin{array}{c}
\text { canonical } \\
\text { topol. map }
\end{array}}{\frac{c_{L / K}}{\longrightarrow}} U_{\widetilde{\mathbb{X}}(L / K)}^{\diamond} / Y_{L / K}
$$

is a bijection with the extended Hazewinkel map $H_{L / K}^{(\varphi)}: U_{\widetilde{\mathbb{X}}(L / K)}^{\diamond} / Y_{L / K} \rightarrow \operatorname{Gal}(L / K)$ as the inverse.

Now, we shall briefly review the constructions of the topological group $Y_{L / K}$ and the extended Hazewinkel map $H_{L / K}^{(\varphi)}: U_{\widetilde{\mathbb{X}}(L / K)}^{\diamond} / Y_{L / K} \rightarrow \operatorname{Gal}(L / K)$. For the details, we refer the reader to the papers [1, 2, 3, which we follow closely.

We fix a basic ascending chain

$$
K=K_{o} \subset K_{1} \subset \cdots \subset K_{i} \subset \cdots \subset L
$$

of subextensions in $L / K$ once and for all. Now, we introduce the following notation. For each $1 \leq i \in \mathbb{Z}$,

(i) let $\sigma_{i}$ be an element of $\operatorname{Gal}(\widetilde{L} / \widetilde{K})$ satisfying $\left\langle\left.\sigma_{i}\right|_{K_{i}}\right\rangle=\operatorname{Gal}\left(K_{i} / K_{i-1}\right)$;

(ii) let $\widetilde{K}_{i}=K_{i} \widetilde{K}$.

By Abelian local class field theory, for each $1 \leq k \in \mathbb{Z}$ we have an injective homomorphism

$$
\Xi_{K_{k+1} / K_{k}}: \operatorname{Gal}\left(K_{k+1} / K_{k}\right) \rightarrow U_{\widetilde{K}_{k+1}} / U_{\widetilde{K}_{k+1}}^{\sigma_{k+1}-1}
$$

defined by

$$
\Xi_{K_{k+1} / K_{k}}: \tau \mapsto \pi_{K_{k+1}}^{\tau-1} U_{\widetilde{K}_{k+1}}^{\sigma_{k+1}-1}
$$

for every $\tau \in \operatorname{Gal}\left(K_{k+1} / K_{k}\right)$. Let $\operatorname{im}\left(\Xi_{K_{k+1} / K_{k}}\right)=T_{k}^{(L / K)}=T_{k}$ be the isomorphic copy of $\operatorname{Gal}\left(K_{k+1} / K_{k}\right)$ in $U_{\widetilde{K}_{k+1}} / U_{\widetilde{K}_{k+1}}^{\sigma_{k+1}-1}$.

Theorem 5.14 (Fesenko). Fix $1 \leq k \in \mathbb{Z}$. Let

$$
T_{k}^{(L / K)^{\prime}}=T_{k}^{\prime}=T_{k} \cap\left(\prod_{1 \leq i \leq k+1} U_{\widetilde{K}_{k+1}}^{\sigma_{i}-1}\right) / U_{\widetilde{K}_{k+1}}^{\sigma_{k+1}-1}
$$


Then the exact sequence

$$
1 \longrightarrow T_{k}^{\prime} \longrightarrow\left(\prod_{1 \leq i \leq k+1} U_{\widetilde{K}_{k+1}}^{\sigma_{i}-1}\right) / \overbrace{\widetilde{K}_{k+1}}^{\sigma_{k+1}-1} \stackrel{\widetilde{N}_{K_{k+1} / K_{k}}}{\longrightarrow} \prod_{1 \leq i \leq k} U_{\widetilde{K}_{k}}^{\sigma_{i}-1} \longrightarrow 1
$$

splits by a homomorphism

$$
h_{k}: \prod_{1 \leq i \leq k} U_{\widetilde{K}_{k}}^{\sigma_{i}-1} \rightarrow\left(\prod_{1 \leq i \leq k+1} U_{\widetilde{K}_{k+1}}^{\sigma_{i}-1}\right) / U_{\widetilde{K}_{k+1}}^{\sigma_{k+1}-1} .
$$

This homomorphism is not unique in general.

For each $1 \leq k \in \mathbb{Z}$, consider any map

$$
g_{k}^{(L / K)}=g_{k}: \prod_{1 \leq i \leq k} U_{\widetilde{K}_{k}}^{\sigma_{i}-1} \rightarrow \prod_{1 \leq i \leq k+1} U_{\widetilde{K}_{k+1}}^{\sigma_{i}-1}
$$

that makes the triangle

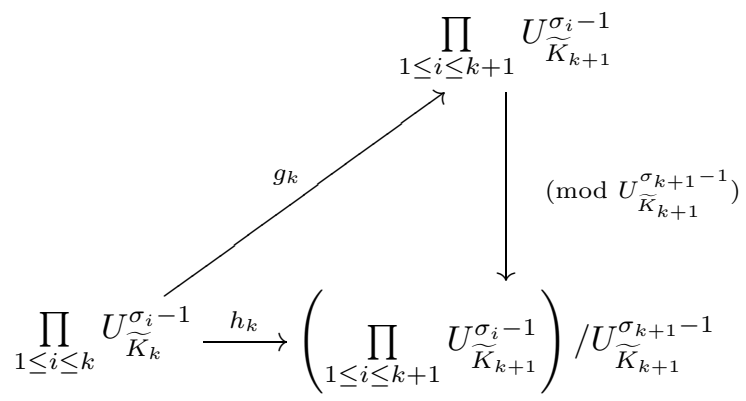

commutative. Clearly, such a map exists. Now, for every $1 \leq i \in \mathbb{Z}$, we choose a mapping

$$
f_{i}^{(L / K)}=f_{i}: U_{\widetilde{K}_{i}}^{\sigma_{i}-1} \rightarrow U_{\widetilde{\mathbb{X}}\left(L / K_{i}\right)} \stackrel{\Lambda_{K_{i} / K}}{\longrightarrow} U_{\widetilde{\mathbb{X}}(L / K)}
$$

such that

$$
\operatorname{Pr}_{\widetilde{K}_{j}} \circ f_{i}=\left.\left(g_{j-1} \circ \cdots \circ g_{i}\right)\right|_{U_{\widetilde{K}_{i}}^{\sigma_{i}-1}}
$$

for each $j \in \mathbb{Z}_{>i}$, where $\operatorname{Pr}_{\widetilde{K}_{j}}: U_{\widetilde{\mathbb{X}}(L / K)} \rightarrow U_{\widetilde{K}_{j}}$ denotes the projection to the $\widetilde{K}_{j^{-}}$ coordinate.

Lemma 5.15 (Fesenko). (i) Let $z^{(i)} \in \operatorname{im}\left(f_{i}\right)=Z_{i}^{(L / K)}$ for each $1 \leq i \in \mathbb{Z}$. Then the infinite product $\prod_{i} z^{(i)}$ converges to an element $z$ in $U_{\widetilde{\mathbb{X}}(L / K)}^{\diamond}$.

(ii) Let

$$
Z_{L / K}\left(\left\{K_{i}, f_{i}\right\}\right)=\left\{\prod_{1 \leq i \in \mathbb{Z}} z^{(i)}: z^{(i)} \in \operatorname{im}\left(f_{i}\right)\right\} .
$$

Then $Z_{L / K}\left(\left\{K_{i}, f_{i}\right\}\right)$ is a topological subgroup of $U_{\widetilde{\mathbb{X}}(L / K)}$.

Remark 5.16. In fact, $Z_{L / K}\left(\left\{K_{i}, f_{i}\right\}\right)$ is a topological subgroup of $U_{\widetilde{\mathbb{X}}(L / K)}^{1}$. Let $z \in$ $Z_{L / K}\left(\left\{K_{i}, f_{i}\right\}\right)$ and choose $z^{(i)} \in \operatorname{im}\left(f_{i}\right) \subset U_{\widetilde{\mathbb{X}}(L / K)}$ so that $z=\prod_{i} z^{(i)}$. It suffices to show that $\operatorname{Pr}_{\widetilde{K}}\left(z^{(i)}\right)=1_{K}$. For this, let $\alpha^{(i)} \in U_{\widetilde{K}_{i}}^{\sigma_{i}-1}$ be such that $f_{i}\left(\alpha^{(i)}\right)=z^{(i)}$. Thus, $\operatorname{Pr}_{\widetilde{K}_{i}}\left(z^{(i)}\right)=\alpha^{(i)}$. Now, by Hilbert's Theorem 90, it follows that $\widetilde{N}_{K_{i} / K}\left(\alpha^{(i)}\right)=$ $\left(\widetilde{N}_{K_{i-1} / K} \circ \widetilde{N}_{K_{i} / K_{i-1}}\right)\left(\alpha^{(i)}\right)=1_{K}$, which completes the proof. 
Lemma 5.17. For $1 \leq i \in \mathbb{Z}$, let $\sigma=\sigma_{i} \in \operatorname{Gal}(\widetilde{L} / \widetilde{K})$ be such that $\left\langle\sigma \mid K_{i}\right\rangle=$ $\operatorname{Gal}\left(K_{i} / K_{i-1}\right)$. Let $\tau \in \operatorname{Gal}(L / K)$ be viewed as an element of $\operatorname{Gal}(\widetilde{L} / \widetilde{K})$. Then

$$
\left(U_{\widetilde{K}_{i}}^{\sigma-1}\right)^{\tau}=U_{\widetilde{K}_{i}}^{\sigma-1}
$$

Proof. Let $\tau$ be any element of $\operatorname{Gal}(L / K)$. We regard $\tau$ as an element of $\operatorname{Gal}(\widetilde{L} / \widetilde{K})$. Clearly, the conjugate $\tau^{-1} \sigma \tau \in \operatorname{Gal}(\widetilde{L} / \widetilde{K})$ satisfies $\left\langle\left.\tau^{-1} \sigma \tau\right|_{K_{i}}\right\rangle=\operatorname{Gal}\left(K_{i} / K_{i-1}\right)$, because $\left(\tau^{-1} \sigma \tau \mid K_{K_{i}}\right)^{n}=\operatorname{id}_{K_{i}}$ yields $\left(\left.\sigma\right|_{K_{i}}\right)^{n}=\operatorname{id}_{K_{i}}$. Let $0<d \in \mathbb{Z}$ be such that $\left.\tau^{-1} \sigma \tau\right|_{K_{i}}=\left(\left.\sigma\right|_{K_{i}}\right)^{d}=\left.\left(\sigma^{d}\right)\right|_{K_{i}}$. Thus, $\tau^{-1} \sigma \tau \sigma^{-d} \in \operatorname{Gal}\left(\widetilde{L} / \widetilde{K}_{i}\right)$ because $\widetilde{K}_{i}=\widetilde{K} K_{i}$. It follows that

$$
U_{\widetilde{K}_{i}}^{\tau^{-1} \sigma \tau-1}=U_{\widetilde{K}_{i}}^{\sigma^{d}-1} .
$$

Since $U_{\widetilde{K}_{i}}^{\tau^{-1}} \sigma \tau-1=U_{\widetilde{K}_{i}}^{\tau^{-1}(\sigma-1) \tau}=\left(U_{\widetilde{K}_{i}}^{\sigma-1}\right)^{\tau}$, the relation

$$
\left(U_{\widetilde{K}_{i}}^{\sigma-1}\right)^{\tau}=U_{\widetilde{K}_{i}}^{\sigma^{d}-1}
$$

also follows. Now, the inclusion

$$
U_{\widetilde{K}_{i}}^{\sigma^{d}-1} \subseteq U_{\widetilde{K}_{i}}^{\sigma-1}
$$

is clear, because, for $u \in U_{\widetilde{K}_{i}}$,

$$
\frac{u^{\sigma^{d}}}{u}=\frac{\left(u^{\sigma^{d-1}}\right)^{\sigma}}{u^{\sigma^{d-1}}} \ldots \frac{\left(u^{\sigma}\right)^{\sigma}}{u^{\sigma}} \frac{u^{\sigma}}{u} .
$$

Thus, for $\tau \in \operatorname{Gal}(L / K)$ we obtain the inclusion $\left(U_{\widetilde{K}_{i}}^{\sigma-1}\right)^{\tau} \subseteq U_{\widetilde{K}_{i}}^{\sigma-1}$. Hence,

$$
\left(U_{\widetilde{K}_{i}}^{\sigma-1}\right)^{\tau}=U_{\widetilde{K}_{i}}^{\sigma-1}
$$

for $\tau \in \operatorname{Gal}(L / K)$, which completes the proof.

Now, let $\tau \in \operatorname{Gal}(L / K)$. Consider the element $\tau^{-1} \sigma_{i} \tau$ of $\operatorname{Gal}(\widetilde{L} / \widetilde{K})$ for each $1 \leq i \in \mathbb{Z}$. Clearly, $\left\langle\left.\tau^{-1} \sigma_{i} \tau\right|_{K_{i}}\right\rangle=\operatorname{Gal}\left(K_{i} / K_{i-1}\right)$. By Abelian local class field theory and by Lemma 5.17, the square

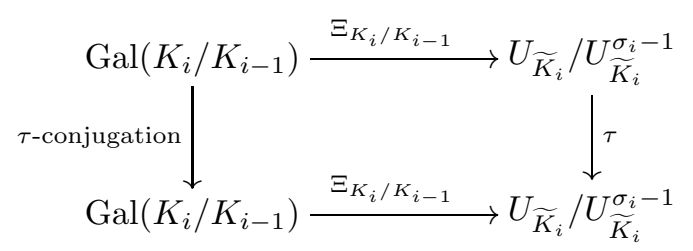

is commutative, where the $\tau$-conjugation map $\operatorname{Gal}\left(K_{i} / K_{i-1}\right) \rightarrow \operatorname{Gal}\left(K_{i} / K_{i-1}\right)$ is defined by $\gamma \mapsto \tau^{-1} \gamma \tau$ for every $\gamma \in \operatorname{Gal}\left(K_{i} / K_{i-1}\right)$. It follows that

$$
\operatorname{im}\left(\Xi_{K_{i} / K_{i-1}}\right)^{\tau}=\operatorname{im}\left(\Xi_{K_{i} / K_{i-1}}\right) .
$$

Now, by Theorem 5.14, for

$$
T_{i}^{\tau}=T_{i}=\operatorname{im}\left(\Xi_{K_{i+1} / K_{i}}\right)
$$

and

$$
\left(T_{i}^{\prime}\right)^{\tau}=T_{i}^{\tau} \cap\left(\prod_{1 \leq j \leq i+1} U_{\widetilde{K}_{i+1}}^{\tau^{-1} \sigma_{j} \tau-1}\right) / U_{\widetilde{K}_{i+1}}^{\tau^{-1}} \sigma_{i+1} \tau-1
$$


the exact sequence

$$
1 \longrightarrow\left(T_{i}^{\prime}\right)^{\tau} \longrightarrow\left(\prod_{1 \leq j \leq i+1} U_{\widetilde{K}_{i+1}^{\tau^{-1}} \sigma_{j} \tau-1}\right) / \overbrace{\widetilde{K}_{i+1}^{\tau^{-1} \sigma_{i+1} \tau-1} \stackrel{\widetilde{N}_{K_{i+1} / K_{i}}}{\longrightarrow} \prod_{1 \leq j \leq i} U_{\widetilde{K}_{i}}^{\tau^{-1} \sigma_{j} \tau-1} \longrightarrow 1}^{\left(h_{i}^{(L / K)}\right)^{\tau}=h_{i}^{\tau}}
$$

splits by a homomorphism

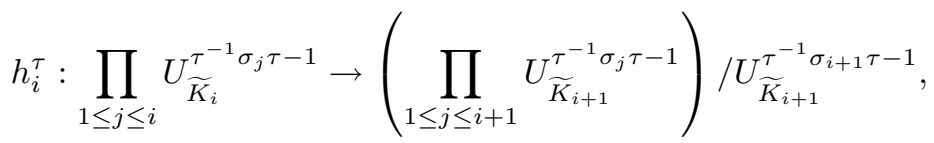

which furthermore makes the diagram

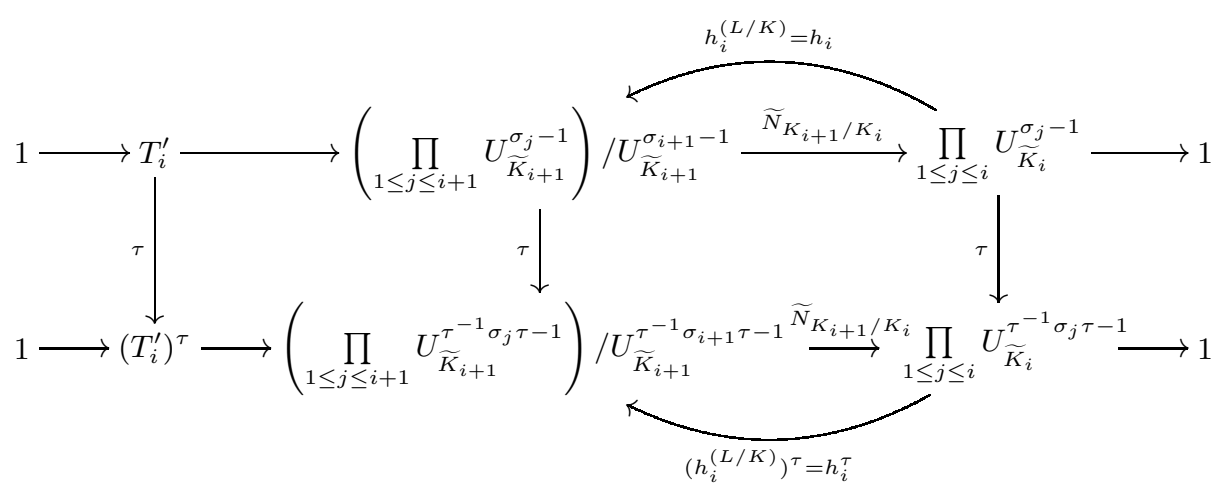

commutative. It follows that there exists a map

$$
g_{i}^{\tau}: \prod_{1 \leq j \leq i} U_{\widetilde{K}_{i}}^{\tau^{-1} \sigma_{j} \tau-1} \rightarrow \prod_{1 \leq j \leq i+1} U_{\widetilde{K}_{i+1}^{\tau^{-1}} \sigma_{j} \tau-1}
$$

that makes the diagram

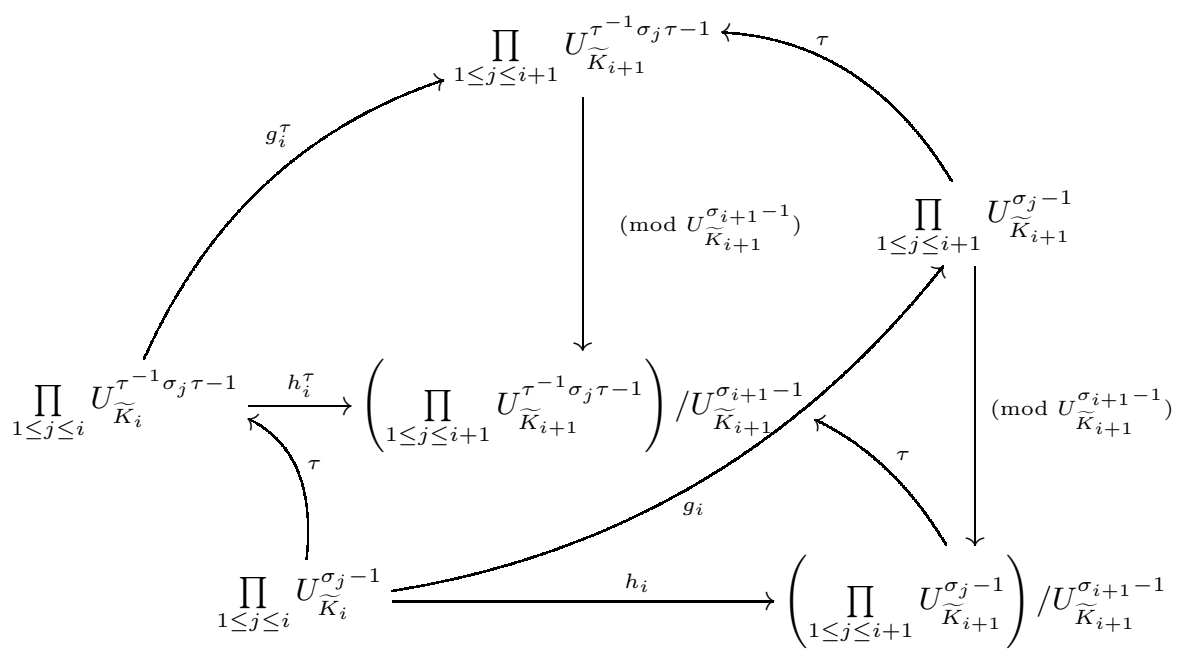

commutative. Now, for every $1 \leq i \in \mathbb{Z}$, choose a mapping

$$
f_{i}^{\tau}: U_{\widetilde{K}_{i}}^{\sigma_{i}-1} \rightarrow U_{\widetilde{\mathbb{X}}(L / K)}
$$

such that

$$
\operatorname{Pr}_{\widetilde{K}_{j}} \circ f_{i}^{\tau}=\left.\left(g_{j-1}^{\tau} \circ \cdots \circ g_{i}^{\tau}\right)\right|_{U_{\widetilde{K}_{i}}^{\sigma_{i}-1}}
$$


for each $j \in \mathbb{Z}_{>i}$. Thus, for $j \in \mathbb{Z}_{>i}$ and $\alpha \in U_{\widetilde{K}_{i}}^{\sigma_{i}-1}$ we have

$$
\operatorname{Pr}_{\widetilde{K}_{j}} \circ f_{i}^{\tau}(\alpha)=\left(\operatorname{Pr}_{\widetilde{K}_{j}} \circ f_{i}\left(\alpha^{\tau^{-1}}\right)\right)^{\tau},
$$

which yields the following relation:

$$
f_{i}^{\tau}(\alpha)=f_{i}\left(\alpha^{\tau^{-1}}\right)^{\tau}
$$

for every $\alpha \in U_{\widetilde{K}_{i}}^{\sigma_{i}-1}$. After all these observations, we state an immediate consequence of Lemma 5.17

Corollary 5.18. For $\tau \in \operatorname{Gal}(L / K)$ we have

$$
Z_{L / K}\left(\left\{K_{i}, f_{i}\right\}\right)^{\tau}=Z_{L / K}\left(\left\{K_{i}, f_{i}^{\tau}\right\}\right) .
$$

Proof. Let $z \in Z_{L / K}\left(\left\{K_{i}, f_{i}\right\}\right)$ and choose $z^{(i)} \in \operatorname{im}\left(f_{i}\right) \subset U_{\widetilde{\mathbb{X}}(L / K)}$ such that $z=$ $\prod_{i} z^{(i)}$. By the continuity of the action of $\operatorname{Gal}(L / K)$ on $U_{\widetilde{\mathbb{X}}(L / K)}$, to prove that $z^{\tau} \in$ $Z_{L / K}\left(\left\{K_{i}, f_{i}^{\tau}\right\}\right)$ it suffices to show that $\left(z^{(i)}\right)^{\tau} \in \operatorname{im}\left(f_{i}^{\tau}\right)$. Now, let $\alpha^{(i)} \in U_{\widetilde{K}_{i}}^{\sigma_{i}-1}$ be such that $f_{i}\left(\alpha^{(i)}\right)=z^{(i)}$. Then $\left(z^{(i)}\right)^{\tau}=f_{i}\left(\alpha^{(i)}\right)^{\tau}=f_{i}\left(\left(\left(\alpha^{(i)}\right)^{\tau}\right)^{\tau^{-1}}\right)^{\tau}=f_{i}^{\tau}\left(\left(\alpha^{(i)}\right)^{\tau}\right)$ by (5.30), where $\left(\alpha^{(i)}\right)^{\tau} \in U_{\widetilde{K}_{i}}^{\sigma_{i}-1}$ by Lemma 5.17, Thus, $\left(z^{(i)}\right)^{\tau} \in \operatorname{im}\left(f_{i}^{\tau}\right)$.

Remark 5.19. By [2, p. 71], if $\tau \in \operatorname{Gal}(L / K)$, then $Z_{L / K}\left(\left\{K_{i}, f_{i}\right\}\right)$ and $Z_{L / K}\left(\left\{K_{i}, f_{i}^{\tau}\right\}\right)$ are algebraically and topologically isomorphic. Thus, Corollary 5.18 indeed defines a continuous action of $\operatorname{Gal}(L / K)$ on $Z_{L / K}\left(\left\{K_{i}, f_{i}\right\}\right)$.

Now, we define the topological subgroup $Y_{L / K}\left(\left\{K_{i}, f_{i}\right\}\right)=Y_{L / K}$ of $U_{\widetilde{\mathbb{X}}(L / K)}^{\diamond}$ to be

$$
Y_{L / K}=\left\{y \in U_{\widetilde{\mathbb{X}}(L / K)}: y^{1-\varphi} \in Z_{L / K}\left(\left\{K_{i}, f_{i}\right\}\right)\right\} .
$$

Lemma 5.20. $Y_{L / K}$ is a topological $\operatorname{Gal}(L / K)$-submodule of $U_{\widetilde{\mathbb{X}}(L / K)}^{\diamond}$.

Proof. Suppose $\tau \in \operatorname{Gal}(L / K)$ and $y \in Y_{L / K}$. Note that $\left(y^{\tau}\right)^{\varphi}=\left(y^{\varphi}\right)^{\tau}$, because the action of $\tau$ on $y=\left(u_{\widetilde{K}_{i}}\right)_{0 \leq i \in \mathbb{Z}}$ is defined by the action of $\tau$ on the " $K_{i}$-part" of $u_{\widetilde{K}_{i}}$ for each $0 \leq i \in \mathbb{Z}$, and the action of $\varphi$ on $y=\left(u_{\widetilde{K}_{i}}\right)_{0 \leq i \in \mathbb{Z}}$ is defined by the action of $\varphi$ on the " $\widetilde{K}$-part" of $u_{\widetilde{K}_{i}}$ for each $0 \leq i \in \mathbb{Z}$. Thus, $\frac{y^{\tau}}{\left(y^{\tau}\right)^{\varphi}}=\frac{y^{\tau}}{\left(y^{\varphi}\right)^{\tau}}=\left(\frac{y}{y^{\varphi}}\right)^{\tau} \in Z_{L / K}\left(\left\{K_{i}, f_{i}\right\}\right)^{\tau}$. Now, the proof follows from Corollary 5.18 and Remark 5.19

Lemma 5.21 (Fesenko). The mapping

$$
\ell_{L / K}^{(\varphi)}: \operatorname{Gal}(L / K) \rightarrow U_{\widetilde{\mathbb{X}}(L / K)}^{1} / Z_{L / K}\left(\left\{K_{i}, f_{i}\right\}\right)
$$

defined by

$$
\ell_{L / K}^{(\varphi)}: \sigma \mapsto \Pi_{\varphi ; L / K}^{\sigma-1} . Z_{L / K}\left(\left\{K_{i}, f_{i}\right\}\right)
$$

for every $\sigma \in \operatorname{Gal}(L / K)$ is a group isomorphism, where the group operation $*$ is defined on $U_{\widetilde{\mathbb{X}}(L / K)}^{1} / Z_{L / K}\left(\left\{K_{i}, f_{i}\right\}\right)$ by

$$
\bar{U} * \bar{V}=\bar{U} \cdot \bar{V}^{\left(\ell_{L / K}^{(\varphi)}\right)^{-1}(\bar{U})}
$$

for every

$$
\bar{U}=U \cdot Z_{L / K}\left(\left\{K_{i}, f_{i}\right\}\right), \quad \bar{V}=V \cdot Z_{L / K}\left(\left\{K_{i}, f_{i}\right\}\right) \in U_{\widetilde{\mathbb{X}}(L / K)}^{1} / Z_{L / K}\left(\left\{K_{i}, f_{i}\right\}\right)
$$

with $U, V \in U_{\widetilde{\mathbb{X}}(L / K)}^{1}$. 
Now, we introduce the fundamental exact sequence

$$
1 \rightarrow \operatorname{Gal}(L / K) \stackrel{\ell_{L / K}^{(\varphi)}}{\longrightarrow} U_{\widetilde{\mathbb{X}}(L / K)} / Z_{L / K}\left(\left\{K_{i}, f_{i}\right\}\right) \stackrel{\operatorname{Pr}_{\widetilde{K}}}{\longrightarrow} U_{\widetilde{K}} \rightarrow 1
$$

as a generalization of the Serre short exact sequence (cf. (1.1) and (1.2)). Thus, for any $U \in U_{\widetilde{\mathbb{X}}(L / K)}^{\diamond}$, since $U^{1-\varphi} \in U_{\widetilde{\mathbb{X}}(L / K)}^{1}$, there exists a unique $\sigma_{U} \in \operatorname{Gal}(L / K)$ satisfying

$$
U^{1-\varphi} \cdot Z_{L / K}\left(\left\{K_{i}, f_{i}\right\}\right)=\ell_{L / K}^{(\varphi)}\left(\sigma_{U}\right),
$$

by Lemma 5.21, Next, define the arrow

$$
H_{L / K}^{(\varphi)}: U_{\widetilde{\mathbb{X}}(L / K)}^{\diamond} / Y_{L / K} \rightarrow \operatorname{Gal}(L / K)
$$

by

$$
H_{L / K}^{(\varphi)}: U . Y_{L / K} \mapsto \sigma_{U}
$$

for every $U \in U_{\widetilde{\mathbb{X}}(L / K)}^{\diamond}$. Clearly, this arrow is a well-defined mapping. Indeed, suppose that $U, V \in U_{\widetilde{\mathbb{X}}(L / K)}^{\diamond}$ satisfy $U \equiv V\left(\bmod Y_{L / K}\right)$. Then $\sigma_{U}=\sigma_{V}$. In fact, let $Y \in$ $Y_{L / K}$ be such that $U=V . Y$. The definition of $Y_{L / K}$ given in (5.31) forces $Y^{1-\varphi} \in$ $Z_{L / K}\left(\left\{K_{i}, f_{i}\right\}\right)$. Since $U^{1-\varphi}=(V . Y)^{1-\varphi}=V^{1-\varphi} Y^{1-\varphi}$, we have $U^{1-\varphi} Z_{L / K}\left(\left\{K_{i}, f_{i}\right\}\right)=$ $V^{1-\varphi} Z_{L / K}\left(\left\{K_{i}, f_{i}\right\}\right)$, which shows that $\ell_{L / K}^{(\varphi)}\left(\sigma_{U}\right)=\ell_{L / K}^{(\varphi)}\left(\sigma_{V}\right)$ by (5.32). Then Lemma 5.21 shows that $\sigma_{U}=\sigma_{V}$.

Lemma 5.22. Suppose that the local field $K$ satisfies condition (5.26). The arrow

$$
H_{L / K}^{(\varphi)}: U_{\widetilde{\mathbb{X}}(L / K)}^{\diamond} / Y_{L / K} \rightarrow \operatorname{Gal}(L / K)
$$

defined for the extension $L / K$ is a bijection.

Proof. Choose $U, V \in U_{\widetilde{\mathbb{X}}(L / K)}^{\diamond}$ satisfying $H_{L / K}^{(\varphi)}\left(U . Y_{L / K}\right)=H_{L / K}^{(\varphi)}\left(V \cdot Y_{L / K}\right)$. Then $\sigma_{U}=$ $\sigma_{V}$ by the definition (5.34) of the arrow (5.33). Now, (5.32) yields

$$
U^{1-\varphi} \cdot Z_{L / K}\left(\left\{K_{i}, f_{i}\right\}\right)=V^{1-\varphi} \cdot Z_{L / K}\left(\left\{K_{i}, f_{i}\right\}\right),
$$

which proves that $\left(V^{-1} U\right)^{1-\varphi} \in Z_{L / K}\left(\left\{K_{i}, f_{i}\right\}\right)$. The fact that $U \cdot Y_{L / K}=V \cdot Y_{L / K}$ follows immediately from (5.31). Now, we choose any $\sigma \in \operatorname{Gal}(L / K)$. By Theorem 5.5, there exists $U \in U_{\widetilde{\mathbb{X}}(L / K)}^{\diamond}$ unique modulo $U_{\mathbb{X}(L / K)}$ (so unique modulo $Y_{L / K}$ because $U_{\mathbb{X}(L / K)} \subseteq$ $\left.Y_{L / K}\right)$ and such that

$$
\Pi_{\varphi ; L / K}^{\sigma-1} . Z_{L / K}\left(\left\{K_{i}, f_{i}\right\}\right)=U^{1-\varphi} \cdot Z_{L / K}\left(\left\{K_{i}, f_{i}\right\}\right) .
$$

Thus, by Theorem 5.21 and (5.32),

$$
\ell_{L / K}^{(\varphi)}(\sigma)=\ell_{L / K}^{(\varphi)}\left(\sigma_{U}\right)
$$

which implies that $\sigma=\sigma_{U}$ for $U \in U_{\widetilde{\mathbb{X}}(L / K)}^{\diamond}$.

Next, consider the composition

$$
\Phi_{L / K}^{(\varphi)}: \operatorname{Gal}(L / K) \stackrel{\phi_{L / K}^{(\varphi)}}{\longrightarrow} U_{\widetilde{\mathbb{X}}(L / K)}^{\diamond} / U_{\mathbb{X}(L / K)} \stackrel{c_{L / K}}{\longrightarrow} U_{\widetilde{\mathbb{X}}(L / K)}^{\diamond} / Y_{L / K} .
$$

Lemma 5.23. (i) $U_{\sigma_{U}} \cdot Y_{L / K}=U . Y_{L / K}$ for every $U \in U_{\widetilde{\mathbb{X}}(L / K)}^{\diamond}$;

(ii) $\sigma_{U_{\sigma}}=\sigma$ for every $\sigma \in \operatorname{Gal}(L / K)$. 
Proof. To prove (i), let $U \in U_{\widetilde{\mathbb{X}}(L / K)}^{\diamond}$. Then, by (15.32), there exists a unique $\sigma_{U} \in$ $\operatorname{Gal}(L / K)$ satisfying

$$
U^{1-\varphi} \cdot Z_{L / K}\left(\left\{K_{i}, f_{i}\right\}\right)=\ell_{L / K}^{(\varphi)}\left(\sigma_{U}\right)=\Pi_{\varphi ; L / K}^{\sigma_{U}-1} \cdot Z_{L / K}\left(\left\{K_{i}, f_{i}\right\}\right) .
$$

The identity on the right-hand side follows from the definition of the mapping $\ell_{L / K}^{(\varphi)}$ : $\operatorname{Gal}(L / K) \rightarrow U_{\widetilde{\mathbb{X}}(L / K)}^{1} / Z_{L / K}\left(\left\{K_{i}, f_{i}\right\}\right)$ given in Lemma 5.21, Now, by Lemma 5.5, for this $\sigma_{U} \in \operatorname{Gal}(L / K)$ there exists $U_{\sigma_{U}} \in U_{\widetilde{\mathbb{X}}(L / K)}^{\diamond}$ (which is unique modulo $U_{\mathbb{X}(L / K)}$ ) satisfying

$$
U_{\sigma_{U}}^{1-\varphi}=\Pi_{\varphi ; L / K}^{\sigma_{U}-1}
$$

Thus,

$$
U_{\sigma_{U}}^{1-\varphi} \cdot Z_{L / K}\left(\left\{K_{i}, f_{i}\right\}\right)=U^{1-\varphi} \cdot Z_{L / K}\left(\left\{K_{i}, f_{i}\right\}\right),
$$

by (5.36), which proves that

$$
U_{\sigma_{U}} . Y_{L / K}=U \cdot Y_{L / K}
$$

by the definition of $Y_{L / K}$ given in (5.31). Moreover, since $U_{\mathbb{X}(L / K)} \subseteq Y_{L / K}$, relation (5.37) does not depend on the choice of $U_{\sigma_{U}}$ modulo $U_{\mathbb{X}(L / K)}$. Now, for (ii), let $\sigma \in \operatorname{Gal}(L / K)$. By Lemma 5.5, there exists $U_{\sigma} \in U_{\widetilde{\mathbb{X}}(L / K)}$ (which is unique modulo $U_{\mathbb{X}(L / K)}$ ) such that

$$
U_{\sigma}^{1-\varphi}=\Pi_{\varphi ; L / K}^{\sigma-1} .
$$

For any such $U_{\sigma} \in U_{\widetilde{\mathbb{X}}(L / K)}^{\diamond}$, there exists a unique $\sigma_{U_{\sigma}} \in \operatorname{Gal}(L / K)$ satisfying

$$
U_{\sigma}^{1-\varphi} \cdot Z_{L / K}\left(\left\{K_{i}, f_{i}\right\}\right)=\ell_{L / K}^{(\varphi)}\left(\sigma_{U_{\sigma}}\right)
$$

by (5.32). Thus, by (5.38) and Lemma 5.21, it follows that

$$
\ell_{L / K}^{(\varphi)}\left(\sigma_{U_{\sigma}}\right)=\Pi_{\varphi ; L / K}^{\sigma-1} . Z_{L / K}\left(\left\{K_{i}, f_{i}\right\}\right)=\ell_{L / K}^{(\varphi)}(\sigma),
$$

which proves that $\sigma_{U_{\sigma}}=\sigma$.

Lemma 5.23 immediately yields

$$
H_{L / K}^{(\varphi)} \circ \Phi_{L / K}^{(\varphi)}=\operatorname{id}_{\mathrm{Gal}(L / K)},
$$

and

$$
\Phi_{L / K}^{(\varphi)} \circ H_{L / K}^{(\varphi)}=\operatorname{id}_{U_{\widetilde{\mathbb{X}}(L / K)}^{\diamond} / Y_{L / K}} .
$$

The following theorem is a consequence of Lemma 5.22 . Lemma 5.23, Theorem 5.6. and the fact that $U_{\mathbb{X}(L / K)}$ is a topological $\mathrm{Gal}(L / K)$-submodule of $Y_{L / K}$.

Theorem 5.24 (Fesenko). Suppose that the local field $K$ satisfies (5.26). The mapping

$$
\Phi_{L / K}^{(\varphi)}: \operatorname{Gal}(L / K) \rightarrow U_{\widetilde{\mathbb{X}}(L / K)}^{\diamond} / Y_{L / K}
$$

defined for the extension $L / K$ is a bijection with the inverse

$$
H_{L / K}^{(\varphi)}: U_{\widetilde{\mathbb{X}}(L / K)}^{\diamond} / Y_{L / K} \rightarrow \operatorname{Gal}(L / K) .
$$

For every $\sigma, \tau \in \operatorname{Gal}(L / K)$ we have

$$
\Phi_{L / K}^{(\varphi)}(\sigma \tau)=\Phi_{L / K}^{(\varphi)}(\sigma) \Phi_{L / K}^{(\varphi)}(\tau)^{\sigma},
$$

i.e., the cocycle condition is satisfied.

By Corollary 5.7, Theorem 5.24 has the following consequence. 
Corollary 5.25. Define a law of composition $*$ on $U_{\widetilde{\mathbb{X}}(L / K)}^{\diamond} / Y_{L / K}$ by

$$
\bar{U} * \bar{V}=\bar{U} \cdot \bar{V}^{\left(\Phi_{L / K}^{(\varphi)}\right)^{-1}(\bar{U})}
$$

for every $\bar{U}=U \cdot Y_{L / K}, \bar{V}=V \cdot Y_{L / K} \in U_{\widetilde{\mathbb{X}}(L / K)}^{\diamond} / Y_{L / K}$ with $U, V \in U_{\widetilde{\mathbb{X}}(L / K)}^{\diamond}$. Then $U_{\widetilde{\mathbb{X}}(L / K)}^{\diamond} / Y_{L / K}$ is a topological group under $*$, and the map $\Phi_{L / K}^{(\varphi)}$ induces an isomorphism of topological groups

$$
\Phi_{L / K}^{(\varphi)}: \operatorname{Gal}(L / K) \stackrel{\sim}{\longrightarrow} U_{\widetilde{\mathbb{X}}(L / K)}^{\diamond} / Y_{L / K},
$$

where the topological group structure on $U_{\widetilde{\mathbb{X}}(L / K)}^{\diamond} / Y_{L / K}$ is defined with respect to the binary operation $*$ given by (5.40).

Definition 5.26. Let $K$ be a local field satisfying (5.26). Let $L / K$ be a totally ramified APF Galois extension satisfying (5.1). The mapping

$$
\Phi_{L / K}^{(\varphi)}: \operatorname{Gal}(L / K) \rightarrow U_{\widetilde{\mathbb{X}}(L / K)}^{\diamond} / Y_{L / K}
$$

defined in Theorem 5.24 is called the Fesenko reciprocity map for the extension $L / K$.

For each $0 \leq i \in \mathbb{R}$, previously we have introduced the groups $\left(U_{\widetilde{\mathbb{X}}(L / K)}^{\diamond}\right)^{i}$. For $0 \leq n \in \mathbb{Z}$, let

$$
Q_{L / K}^{n}=c_{L / K}\left(\left(U_{\widetilde{\mathbb{X}}(L / K)}^{\diamond}\right)^{n} U_{\mathbb{X}(L / K)} / U_{\mathbb{X}(L / K)} \cap \operatorname{im}\left(\phi_{L / K}^{(\varphi)}\right)\right),
$$

which is a subgroup of $\left(U_{\widetilde{\mathbb{X}}(L / K)}^{\diamond}\right)^{n} Y_{L / K} / Y_{L / K}$. Now, the Fesenko ramification theorem, stated in Theorem 5.8, can be reformulated for the reciprocity map $\Phi_{L / K}^{(\varphi)}$ corresponding to the extension $L / K$ as follows.

Theorem 5.27 (Ramification theorem). Suppose that the local field $K$ satisfies the condition given in (5.26). For $0 \leq n \in \mathbb{Z}$, let $\mathrm{Gal}(L / K)_{n}$ denote the $n$th higher ramification subgroup of the Galois group $\operatorname{Gal}(L / K)$ corresponding to the APF Galois subextension $L / K$ of $K_{\varphi} / K$ in the lower numbering. Then we have the inclusion

$$
\Phi_{L / K}^{(\varphi)}\left(\operatorname{Gal}(L / K)_{n}-\operatorname{Gal}(L / K)_{n+1}\right) \subseteq\left(U_{\widetilde{\mathbb{X}}(L / K)}^{\diamond}\right)^{n} Y_{L / K} / Y_{L / K}-Q_{L / K}^{n+1} .
$$

Proof. Let $\tau \in \operatorname{Gal}(L / K)_{n}$. The first half of the proof of Proposition 1 in 2] shows that $\Phi_{L / K}^{(\varphi)}(\tau) \in\left(U_{\widetilde{\mathbb{X}}(L / K)}^{\diamond}\right)^{n} Y_{L / K} / Y_{L / K}$. Now, let $\bar{U}=U . Y_{L / K} \in Q_{L / K}^{n+1}$, where $U \in$ $U_{\widetilde{\mathbb{X}}(L / K)}^{\diamond}$. Then, by the definition of $Q_{L / K}^{n+1}$, there exist $V \in\left(U_{\widetilde{\mathbb{X}}(L / K)}^{\diamond}\right)^{n+1} U_{\mathbb{X}(L / K)}$ and $\tau \in \operatorname{Gal}(L / K)$ such that $c_{L / K}(\bar{V})=\bar{U}$ and $\phi_{L / K}^{(\varphi)}(\tau)=\bar{V}$, where $\bar{V}=V \cdot U_{\mathbb{X}(L / K)}$. So, $\Phi_{L / K}^{(\varphi)}(\tau)=\bar{U}$. The second half of the proof of Proposition 1 in [2] now proves that $\tau \in \operatorname{Gal}(L / K)_{n+1}$.

Let $M / K$ be a Galois subextension of $L / K$. Thus, there exists a chain of field extensions

$$
K \subseteq M \subseteq L \subseteq K_{\varphi}
$$

where $M$ is a totally ramified APF Galois extension over the local field $K$ satisfying (5.26) by Lemma 3.3 . $M$,

The basic ascending chain of subextensions in $L / K$ fixed in (5.27) and restricted to

$$
K=K_{o} \cap M \subseteq K_{1} \cap M \subseteq \cdots \subseteq K_{i} \cap M \subseteq \cdots \subseteq L \cap M=M
$$


is almost a basic ascending chain of subextensions in $M / K$ (in the sense that there may exist elements $0 \leq i \in \mathbb{Z}$ such that $\left.K_{i} \cap M=K_{i+1} \cap M\right)$. In fact, for each $0 \leq i \in \mathbb{Z}$, the extension $K_{i} \cap M / K$ is clearly Galois. For each $0 \leq i \in \mathbb{Z}$, consider the surjective homomorphism

$$
r_{K_{i+1} \cap M}: \operatorname{Gal}\left(K_{i+1} / K_{i}\right) \rightarrow \operatorname{Gal}\left(K_{i+1} \cap M / K_{i} \cap M\right)
$$

defined by restriction to $K_{i+1} \cap M$,

$$
\left.\sigma \mapsto \sigma\right|_{K_{i+1} \cap M},
$$

for every $\sigma \in \operatorname{Gal}\left(K_{i+1} / K_{i}\right)$. Since $\operatorname{Gal}\left(K_{i+1} / K_{i}\right)$ is cyclic of prime order $p=\operatorname{char}\left(\kappa_{K}\right)$ (respectively, of order relatively prime to $p$ ) for $1 \leq i \in \mathbb{Z}$ (respectively, for $i=0$ ), it follows that $\operatorname{Gal}\left(K_{i+1} \cap M / K_{i} \cap M\right)$ is cyclic of order $p$ or 1 (respectively, of order relatively prime to $p$ ) for $1 \leq i \in \mathbb{Z}$ (respectively, for $i=0$ ). Now we fix this almost basic ascending chain of subextensions in $M / K$, introduced in (5.43). Observe that, for each $1 \leq i \in \mathbb{Z},\left.\sigma_{i}\right|_{\widetilde{M}} \in \operatorname{Gal}(\widetilde{M} / \widetilde{K})$ satisfies

$$
\left\langle\left.\left(\left.\sigma_{i}\right|_{\widetilde{M}}\right)\right|_{K_{i} \cap M}=\left.\sigma_{i}\right|_{K_{i} \cap M}\right\rangle=\operatorname{Gal}\left(K_{i} \cap M / K_{i-1} \cap M\right),
$$

because the restriction map $r_{K_{i} \cap M}: \operatorname{Gal}\left(K_{i} / K_{i-1}\right) \rightarrow \operatorname{Gal}\left(K_{i} \cap M / K_{i-1} \cap M\right)$ is a surjective homomorphism and $\left\langle\left.\sigma_{i}\right|_{K_{i}}\right\rangle=\operatorname{Gal}\left(K_{i} / K_{i-1}\right)$. As usual, we set $\widehat{K_{i} \cap M}=$ $\left(K_{i} \cap M\right) \widetilde{K}$. Note that, for each $1 \leq k \in \mathbb{Z}$, the norm map

$$
\widetilde{N}_{K_{k+1} / K_{k+1} \cap M}: U_{\widetilde{K}_{k+1}} \rightarrow U_{K_{k+1} \cap M}
$$

induces a homomorphism

$$
\widetilde{N}_{K_{k+1} / K_{k+1} \cap M}^{*}: U_{\widetilde{K}_{k+1}} / U_{\widetilde{K}_{k+1}}^{\sigma_{k+1}-1} \rightarrow U_{K_{k+1} \cap M} / U_{K_{k+1} \cap M}^{\left.\sigma_{k+1}\right|_{\widetilde{M}}-1}
$$

defined by

$$
\widetilde{N}_{K_{k+1} / K_{k+1} \cap M}^{*}: u \cdot U_{\widetilde{K}_{k+1}^{\sigma_{k+1}-1}}^{\sigma_{N}} \widetilde{N}_{K_{k+1} / K_{k+1} \cap M}(u) \cdot U_{K_{k+1} \cap M}^{\left.\sigma_{k+1}\right|_{\widetilde{M}}-1}
$$

for every $u \in U_{\widetilde{K}_{k+1}}$, because $\widetilde{N}_{K_{k+1} / K_{k+1} \cap M}\left(U_{\widetilde{K}_{k+1}}^{\sigma_{k+1}-1}\right) \subseteq U_{K_{k+1} \cap M}^{\left.\sigma_{k+1}\right|_{\widetilde{M}}-1}$. Thus, the following square (in which) the upper and lower horizontal arrows are defined by (5.28) and (5.29),

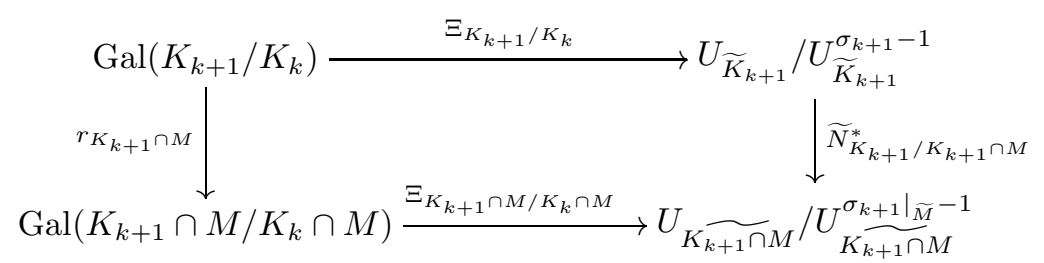

is commutative, because $\tilde{N}_{K_{k+1} / K_{k+1} \cap M}\left(\pi_{K_{k+1}}\right)=\pi_{K_{k+1} \cap M}$ by the norm coherence of the Lubin-Tate labelling $\left(\pi_{K^{\prime}}\right)_{K \subseteq K^{\prime} \subset K_{\varphi}}$, where $\left[K^{\prime}: K\right]<\infty$. Hence,

$$
\widetilde{N}_{K_{k+1} / K_{k+1} \cap M}\left(T_{k}^{(L / K)}\right)=T_{k}^{(M / K)} .
$$

Next, we define an arrow

$$
h_{k}^{(M / K)}: \prod_{1 \leq i \leq k} U^{\left.\sigma_{i}\right|_{\widetilde{M}}-1} \rightarrow\left(\prod_{K_{k} \cap M} U_{1 \leq i \leq k+1}^{\left.\sigma_{i}\right|_{\widetilde{M}}-1}\right) / U_{K_{k+1} \cap M}^{\left.\sigma_{k+1}\right|_{\widetilde{M}}-1}
$$


that splits the exact sequence

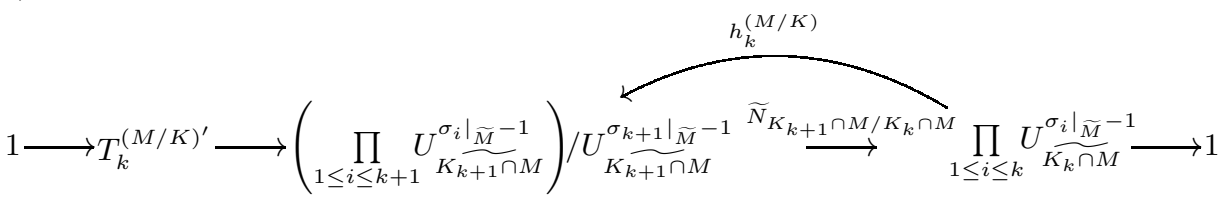

in such a way that

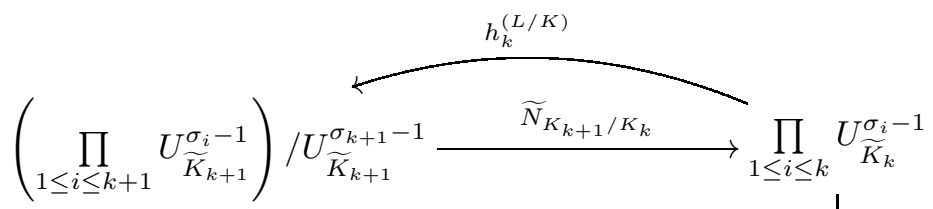

$$
\begin{aligned}
& \widetilde{N}_{K_{k+1} / K_{k+1} \cap M}^{*} \downarrow \mid \widetilde{N}_{K_{k} / K_{k} \cap M}
\end{aligned}
$$

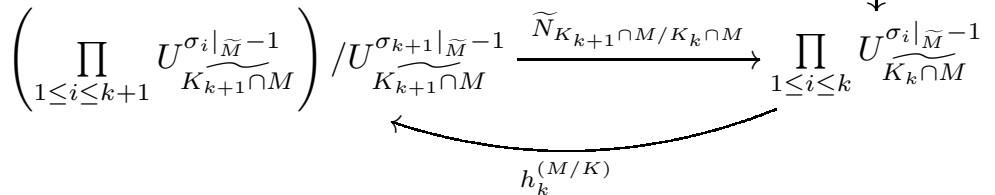

is a commutative square. For this, however, closely following Fesenko (see [1, 2, 3]), we review the construction of a splitting

$$
h_{k}^{(L / K)}: \prod_{1 \leq i \leq k} U_{\widetilde{K}_{k}}^{\sigma_{i}-1} \rightarrow\left(\prod_{1 \leq i \leq k+1} U_{\widetilde{K}_{k+1}^{\sigma_{i}-1}}^{\sigma^{2}}\right) / U_{\widetilde{K}_{k+1}}^{\sigma_{k+1}-1}
$$

of the short exact sequence

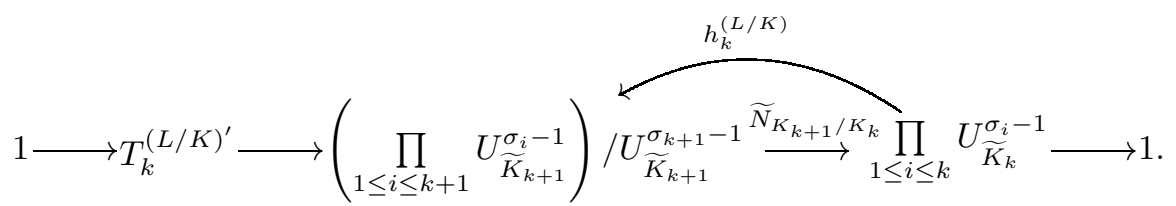

The product module $\prod_{1 \leq i \leq k} U_{\widetilde{K}_{k}}^{\sigma_{i}-1}$ is a closed $\mathbb{Z}_{p}$-submodule of $U_{\widetilde{K}_{k}}^{1}$. Let $\left\{\lambda_{j}\right\}$ be a system of topological multiplicative generators of the topological $\mathbb{Z}_{p}$-module $\prod_{1 \leq i \leq k} U_{\widetilde{K}_{k}}^{\sigma_{i}-1}$ satisfying the following property. If the torsion of the module $\prod_{1 \leq i \leq k} U_{\widetilde{K}_{k}}^{\sigma_{i}-1}$ is nontrivial, there exists $\lambda_{*} \in\left\{\lambda_{j}\right\}$ of order $p^{m}$ in the torsion of the module, while the remaining $\lambda_{j}$ $(j \neq *)$ are topologically independent over $\mathbb{Z}_{p}$. Now, we define a map

$$
h_{k}^{(L / K)}:\left\{\lambda_{j}\right\} \rightarrow\left(\prod_{1 \leq i \leq k+1} U_{\widetilde{K}_{k+1}^{\sigma_{i}-1}}\right) / U_{\widetilde{K}_{k+1}^{\sigma_{k+1}-1}}
$$

on the topological generators $\left\{\lambda_{j}\right\}$ by

$$
h_{k}^{(L / K)}: \lambda_{j} \mapsto u_{j} . U_{\widetilde{K}_{k+1}}^{\sigma_{k+1}-1},
$$

where $u_{j} \in \prod_{1 \leq i \leq k+1} U_{\widetilde{K}_{k+1}}^{\sigma_{i}-1}$ satisfies $\widetilde{N}_{K_{k+1} / K_{k}}\left(u_{j}\right)=\lambda_{j}$. By step 5 of the proof of the theorem in $\S 3$ of [3], it follows that $h_{k}^{(L / K)}\left(\lambda_{*}\right)^{p^{m}} \in U_{\widetilde{K}_{k+1}}^{\sigma_{k+1}-1}$. Therefore, the arrow 
$h_{k}^{(L / K)}$ extends uniquely to a homomorphism

$$
h_{k}^{(L / K)}: \prod_{1 \leq i \leq k} U_{\widetilde{K}_{k}}^{\sigma_{i}-1} \rightarrow\left(\prod_{1 \leq i \leq k+1} U_{\widetilde{K}_{k+1}^{\sigma_{i}-1}}\right) / U_{\widetilde{K}_{k+1}}^{\sigma_{k+1}-1},
$$

which is a splitting of the short exact sequence (5.47). Now, we define

$$
h_{k}^{(M / K)}: \prod_{1 \leq i \leq k} U_{\frac{\left.K_{k} \cap\right|_{\widetilde{M}}-1}{K_{k} \cap M}}^{\rightarrow}\left(\prod_{1 \leq i \leq k+1} U_{K_{k+1} \cap M}^{\left.\sigma_{i}\right|_{\widetilde{M}}-1}\right) / U_{K_{k+1} \cap M}^{\left.\sigma_{k+1}\right|_{\widetilde{M}}-1}
$$

as follows. Observe that

$$
\widetilde{N}_{K_{k} / K_{k} \cap M}: \prod_{1 \leq i \leq k} U_{\widetilde{K}_{k}}^{\sigma_{i}-1} \rightarrow \prod_{1 \leq i \leq k} U_{K_{k} \cap M}^{\left.\sigma_{i}\right|_{\widetilde{M}}-1}
$$

is a surjective homomorphism, because $\widetilde{N}_{K_{k} / K_{k} \cap M}: U_{\widetilde{K}_{k}} \rightarrow \widetilde{U_{\overparen{K_{k}} \cap M}}$ is a surjective homomorphism. Thus, the collection $\left\{\widetilde{N}_{K_{k} / K_{k} \cap M}\left(\lambda_{j}\right)\right\}$ is a system of topological multiplicative generators of the topological $\mathbb{Z}_{p}$-module $\prod_{1 \leq i \leq k} U_{\widetilde{K_{k} \cap M}}^{\left.\sigma_{i}\right|_{\widetilde{M}}-1}$. Moreover, note that $\widetilde{N}_{K_{k} / K_{k} \cap M}\left(\lambda_{*}\right)^{p^{m}}=1$. Therefore, $\widetilde{N}_{K_{k} / K_{k} \cap M}\left(\lambda_{*}\right)$ is contained in the torsion part $\left(\prod_{1 \leq i \leq k} U_{\widetilde{K_{k} \cap M}}^{\left.\sigma_{i}\right|_{\widetilde{M}}-1}\right)_{\text {tor }}$. For the remaining $\lambda_{j}(j \neq *)$, the collection $\left\{\widetilde{N}_{K_{k} / K_{k} \cap M}\left(\lambda_{j}\right)\right\}_{j \neq *}$ is topologically independent over $\mathbb{Z}_{p}$. Now, following Fesenko's construction of $h_{k}^{(L / K)}$, we define a map

$$
h_{k}^{(M / K)}:\left\{\widetilde{N}_{K_{k} / K_{k} \cap M}\left(\lambda_{j}\right)\right\} \rightarrow\left(\prod_{1 \leq i \leq k+1} U_{K_{k+1}^{\left.\sigma_{i}\right|_{\widetilde{M}}-1}}^{\widetilde{r} M}\right) / U_{\left.K_{k+1} \sigma_{k+1}\right|_{\widetilde{M}}-1}^{\widetilde{M}}
$$

on the topological generators $\left\{\widetilde{N}_{K_{k} / K_{k} \cap M}\left(\lambda_{j}\right)\right\}$ by

$$
h_{k}^{(M / K)}: \widetilde{N}_{K_{k} / K_{k} \cap M}\left(\lambda_{j}\right) \mapsto \widetilde{N}_{K_{k+1} / K_{k+1} \cap M}\left(u_{j}\right) \cdot U_{\left.K_{k+1} \sigma_{k+1}\right|_{\widetilde{M}}-1},
$$

where $u_{j} \in \prod_{1 \leq i \leq k+1} U_{\widetilde{K}_{k+1}}^{\sigma_{i}-1}$ satisfies $\widetilde{N}_{K_{k+1} / K_{k}}\left(u_{j}\right)=\lambda_{j}$, and $\widetilde{N}_{K_{k+1} / K_{k+1} \cap M}\left(u_{j}\right)$ satisfies

$$
\begin{gathered}
\widetilde{N}_{K_{k+1} \cap M / K_{k} \cap M}\left(\widetilde{N}_{K_{k+1} / K_{k+1} \cap M}\left(u_{j}\right)\right)=\widetilde{N}_{K_{k+1} / K_{k} \cap M}\left(u_{j}\right) \\
=\widetilde{N}_{K_{k} / K_{k} \cap M}\left(\widetilde{N}_{K_{k+1} / K_{k}}\left(u_{j}\right)\right)=\widetilde{N}_{K_{k} / K_{k} \cap M}\left(\lambda_{j}\right) .
\end{gathered}
$$

Therefore, the arrow $h_{k}^{(M / K)}$ extends uniquely to a homomorphism

$$
h_{k}^{(M / K)}: \prod_{1 \leq i \leq k} U^{\left.\sigma_{i}\right|_{\widetilde{M}}-1} \rightarrow\left(\prod_{1 \leq i \leq k+1} U_{K_{k} \cap M}^{\left.\sigma_{i}\right|_{\widetilde{M}}-1}\right) / U_{K_{k+1} \cap M}^{\left.\sigma_{k+1}\right|_{\widetilde{M}}-1},
$$

which is a splitting of the short exact sequence given by (5.45). Indeed, it suffices to show that, for $u_{j} \in \prod_{1 \leq i \leq k+1} U_{\widetilde{K}_{k+1}}^{\sigma_{i}-1}$ satisfying $\widetilde{N}_{K_{k+1} / K_{k}}\left(u_{j}\right)=\lambda_{j}$, we have

$$
\begin{aligned}
& h_{k}^{(M / K)} \circ \widetilde{N}_{K_{k+1} \cap M / K_{k} \cap M}: \widetilde{N}_{K_{k+1} / K_{k+1} \cap M}\left(u_{j}\right) U_{\left.K_{k+1} \cap \sigma_{k+1}\right|_{\widetilde{M}}-1} \\
& \mapsto \widetilde{N}_{K_{k+1} / K_{k+1} \cap M}\left(u_{j}\right) U_{K_{k+1} \cap M}^{\left.\sigma_{k+1}\right|_{\widetilde{M}}-1},
\end{aligned}
$$


which follows from the identities

$$
\begin{gathered}
h_{k}^{(M / K)}\left(\widetilde{N}_{K_{k+1} \cap M / K_{k} \cap M}\left(\widetilde{N}_{K_{k+1} / K_{k+1} \cap M}\left(u_{j}\right)\right)\right)=h_{k}^{(M / K)}\left(\widetilde{N}_{K_{k+1} / K_{k} \cap M}\left(u_{j}\right)\right) \\
=h_{k}^{(M / K)}\left(\widetilde{N}_{K_{k} / K_{k} \cap M}\left(\widetilde{N}_{K_{k+1} / K_{k}}\left(u_{j}\right)\right)\right)=h_{k}^{(M / K)}\left(\widetilde{N}_{K_{k} / K_{k} \cap M}\left(\lambda_{j}\right)\right)
\end{gathered}
$$

and from the definition (5.48) of the arrow $h_{k}^{(M / K)}$. Moreover, the diagram (5.46) commutes, because

$$
\begin{aligned}
h_{k}^{(M / K)}\left(\widetilde{N}_{K_{k} / K_{k} \cap M}\left(\lambda_{j}\right)\right) & =\widetilde{N}_{K_{k+1} / K_{k+1} \cap M}\left(u_{j}\right) U_{K_{k+1} \cap M}^{\left.\sigma_{k+1}\right|_{\widetilde{M}^{-}}-1} \\
& =\widetilde{N}_{K_{k+1} / K_{k+1} \cap M}^{*}\left(u_{j} \cdot U_{\widetilde{K}_{k+1}}^{\sigma_{k+1}-1}\right) \\
& =\widetilde{N}_{K_{k+1} / K_{k+1} \cap M}^{*}\left(h_{k}^{(L / K)}\left(\lambda_{j}\right)\right) .
\end{aligned}
$$

For each $1 \leq k \in \mathbb{Z}$, consider any map

$$
g_{k}^{(M / K)}: \prod_{1 \leq i \leq k} U_{\overparen{K_{k} \cap M}}^{\left.\sigma_{i}\right|_{\widetilde{M}}-1} \rightarrow \prod_{1 \leq i \leq k+1} U_{\left.K_{k+1}^{\sigma_{i}}\right|_{\widetilde{M}}-1}
$$

that makes the square

$$
\begin{aligned}
& \prod_{1 \leq i \leq k} U_{\widetilde{K}_{k}}^{\sigma_{i}-1} \stackrel{g_{k}^{(L / K)}}{\longrightarrow} \prod_{1 \leq i \leq k+1} U_{\widetilde{K}_{k+1}}^{\sigma_{i}-1} \\
& \widetilde{N}_{K_{k} / K_{k} \cap M} \downarrow \downarrow \widetilde{N}_{K_{k+1} / K_{k+1} \cap M} \\
& \prod_{1 \leq i \leq k} U_{\widetilde{K_{k} \cap M}}^{\left.\sigma_{i}\right|_{\widetilde{M}}-1} g_{k}^{(M / K)} \prod_{1 \leq i \leq k+1} U_{K_{k+1} \cap M}^{\left.\sigma_{i}\right|_{\widetilde{M}-1}}
\end{aligned}
$$

commutative. Note that such a map satisfies

$$
h_{k}^{(M / K)}=g_{k}^{(M / K)} \bmod U_{\left.K_{k+1} \sigma_{k+1}\right|_{\widetilde{M}}-1}^{\widehat{n}} .
$$

Indeed, by the commutative diagram (5.46), for any $w \in \prod_{1 \leq i \leq k} U_{\widehat{K_{k} \cap M}}^{\left.\sigma_{i}\right|_{\widetilde{M}}-1}$, there exists $v \in \prod_{1 \leq i \leq k} U_{\widetilde{K}_{k}}^{\sigma_{i}-1}$ such that $w=\widetilde{N}_{K_{k} / K_{k} \cap M}(v)$, and

$$
\begin{aligned}
h_{k}^{(M / K)}(w) & =h_{k}^{(M / K)}\left(\widetilde{N}_{K_{k} / K_{k} \cap M}(v)\right) \\
& =\widetilde{N}_{K_{k+1} / K_{k+1} \cap M}^{*}\left(h_{k}^{(L / K)}(v)\right) \\
& =\widetilde{N}_{K_{k+1} / K_{k+1} \cap M}^{*}\left(g_{k}^{(L / K)}(v) \cdot U_{\widetilde{K}_{k+1}}^{\sigma_{k+1}-1}\right) \\
& =\widetilde{N}_{K_{k+1} / K_{k+1} \cap M}\left(g_{k}^{(L / K)}(v)\right) \cdot U_{K_{k+1}^{\sigma_{k+1}} \widetilde{\widetilde{M}}_{\overparen{M}}-1},
\end{aligned}
$$

and by the commutativity of the diagram (5.50) we have

$$
\tilde{N}_{K_{k+1} / K_{k+1} \cap M}\left(g_{k}^{(L / K)}(v)\right)=g_{k}^{(M / K)}\left(\tilde{N}_{K_{k} / K_{k} \cap M}(v)\right)=g_{k}^{(M / K)}(w) .
$$

Thus, the relation

$$
h_{k}^{(M / K)}(w)=g_{k}^{(M / K)}(w) \cdot U_{K_{k+1} \cap M}^{\left.\sigma_{k+1}\right|_{\widetilde{M}}-1}
$$

follows for every $w \in \prod_{1 \leq i \leq k} U_{\widetilde{K_{k} \cap M}}^{\left.\sigma_{i}\right|_{\widetilde{M}}-1}$.

Now, for each $1 \leq i \in \mathbb{Z}$, we introduce the map

$$
f_{i}^{(M / K)}: U_{\widetilde{K_{i} \cap M}}^{\left.\sigma_{i}\right|_{\widetilde{M}}-1} \rightarrow U_{\widetilde{\mathbb{X}}(M / K)}
$$


by

$$
f_{i}^{(M / K)}(w)=\widetilde{\mathcal{N}}_{L / M}\left(f_{i}^{(L / K)}(v)\right)
$$

where $v \in U_{\widetilde{K}_{i}}^{\sigma_{i}-1}$ is any element satisfying $\widetilde{N}_{K_{i} / K_{i} \cap M}(v)=w \in U_{\widetilde{K_{i} \cap M}}^{\left.\sigma_{i}\right|_{\widetilde{M}}-1}$. Note that if $v^{\prime} \in U_{\widetilde{K}_{i}}^{\sigma_{i}-1}$ is such that $\widetilde{N}_{K_{i} / K_{i} \cap M}\left(v^{\prime}\right)=w$, then $\widetilde{\mathcal{N}}_{L / M}\left(f_{i}^{(L / K)}(v)\right)=\widetilde{\mathcal{N}}_{L / M}\left(f_{i}^{(L / K)}\left(v^{\prime}\right)\right)$. In fact, there exists $u \in \operatorname{ker}\left(\widetilde{N}_{K_{i} / K_{i} \cap M}\right)$ such that $v^{\prime}=v u$. Thus, we need to verify that $\widetilde{\mathcal{N}}_{L / M}\left(f_{i}^{(L / K)}(v)\right)=\widetilde{\mathcal{N}}_{L / M}\left(f_{i}^{(L / K)}(v u)\right)$. That is, for each $1 \leq j \in \mathbb{Z}$, we must check that

$$
\widetilde{N}_{K_{j} / K_{j} \cap M}\left(\operatorname{Pr}_{\widetilde{K}_{j}}\left(f_{i}^{(L / K)}(v)\right)\right)=\widetilde{N}_{K_{j} / K_{j} \cap M}\left(\operatorname{Pr}_{\widetilde{K}_{j}}\left(f_{i}^{(L / K)}(v u)\right)\right) .
$$

But for $j>i$ we have

$$
\begin{aligned}
\widetilde{N}_{K_{j} / K_{j} \cap M}\left(\operatorname{Pr}_{\widetilde{K}_{j}}\left(f_{i}^{(L / K)}(v)\right)\right) & =\widetilde{N}_{K_{j} / K_{j} \cap M}\left(g_{j-1}^{(L / K)} \circ \cdots \circ g_{i}^{(L / K)}(v)\right) \\
& =g_{j-1}^{(M / K)} \circ \cdots \circ g_{i}^{(M / K)}\left(\widetilde{N}_{K_{i} / K_{i} \cap M}(v)\right) \\
& =g_{j-1}^{(M / K)} \circ \cdots \circ g_{i}^{(M / K)}\left(\widetilde{N}_{K_{i} / K_{i} \cap M}(v u)\right) \\
& =\widetilde{N}_{K_{j} / K_{j} \cap M}\left(g_{j-1}^{(L / K)} \circ \cdots \circ g_{i}^{(L / K)}(v u)\right) \\
& =\widetilde{N}_{K_{j} / K_{j} \cap M}\left(\operatorname{Pr}_{\widetilde{K}_{j}}\left(f_{i}^{(L / K)}(v u)\right)\right) .
\end{aligned}
$$

Thus, the map

$$
f_{i}^{(M / K)}: U_{\widehat{K_{i} \cap M}}^{\left.\sigma_{i}\right|_{\widetilde{M}}-1} \rightarrow U_{\widetilde{\mathbb{X}}(M / K)}
$$

is well defined. Moreover,

$$
\operatorname{Pr} \widetilde{K_{j} \cap M} \circ f_{i}^{(M / K)}=\left.\left(g_{j-1}^{(M / K)} \circ \cdots \circ g_{i}^{(M / K)}\right)\right|_{U_{\widetilde{K_{i} \cap M}}^{\left.\sigma_{i}\right|_{\widetilde{M}} ^{-1}}}
$$

for $j>i$. Indeed, for $w \in U_{\widehat{K_{i} \cap M}}^{\left.\sigma_{i}\right|_{\widetilde{M}}-1}$, there exists $v \in U_{\widetilde{K}_{i}}^{\sigma_{i}-1}$ with $\widetilde{N}_{K_{i} / K_{i} \cap M}(v)=w$, and $f_{i}^{(M / K)}(w)=\tilde{\mathcal{N}}_{L / M}\left(f_{i}^{(L / K)}(v)\right)$. That is, the square

$$
\begin{aligned}
& U_{\widetilde{K}_{i}}^{\sigma_{i}-1} \stackrel{f_{i}^{(L / K)}}{\longrightarrow} U_{\widetilde{\mathbb{X}}}(L / K) \\
& \left.\widetilde{N}_{K_{i} / K_{i} \cap M}\right|_{\frac{K_{i} \cap M}{\left.\sigma_{i}\right|_{\widetilde{M}}-1} \stackrel{f_{i}^{(M / K)}}{\longrightarrow} U_{\widetilde{\mathbb{X}}(M / K)}} \downarrow \widetilde{\mathcal{N}}_{L / M}
\end{aligned}
$$

is commutative. Thus,

$$
\begin{aligned}
\operatorname{Pr}_{\widetilde{K_{j} \cap M}} \circ f_{i}^{(M / K)}(w) & =\operatorname{Pr}_{\widetilde{K_{j} \cap M}} \circ \widetilde{\mathcal{N}}_{L / M}\left(f_{i}^{(L / K)}(v)\right) \\
& =\widetilde{N}_{K_{j} / K_{j} \cap M}\left(\operatorname{Pr}_{\widetilde{K}_{j}} \circ f_{i}^{(L / K)}(v)\right) \\
& =\widetilde{N}_{K_{j} / K_{j} \cap M}\left(\left(g_{j-1}^{(L / K)} \circ \cdots \circ g_{i}^{(L / K)}\right)(v)\right) \\
& =\left(g_{j-1}^{(M / K)} \circ \cdots \circ g_{i}^{(M / K)}\right)\left(\widetilde{N}_{K_{i} / K_{i} \cap M}(v)\right),
\end{aligned}
$$

as desired.

For each $0<i \in \mathbb{Z}$, let

$$
Z_{i}^{(M / K)}=\operatorname{im}\left(f_{i}^{(M / K)}\right)
$$


Then, by Lemma 5.15$]$ or by [2, Lemma 4], for $z^{(i)} \in Z_{i}^{(M / K)}$ the product $\prod_{i} z^{(i)}$ converges to an element in $U_{\widetilde{\mathbb{X}}(M / K)}^{\diamond}$. Let

$$
Z_{M / K}\left(\left\{K_{i} \cap M, f_{i}^{(M / K)}\right\}\right)=\left\{\prod_{i} z^{(i)}: z^{(i)} \in Z_{i}^{(M / K)}\right\},
$$

which is a topological subgroup of $U_{\widetilde{\mathbb{X}}(M / K)}$. We introduce the topological $\operatorname{Gal}(M / K)$ submodule $Y_{M / K}\left(\left\{K_{i} \cap M, f_{i}^{(M / K)}\right\}\right)=Y_{M / K}$ of $U_{\widetilde{\mathbb{X}}(M / K)}^{\diamond}$ by

$$
Y_{M / K}=\left\{y \in U_{\widetilde{\mathbb{X}}(M / K)}: y^{1-\varphi} \in Z_{M / K}\left(\left\{K_{i} \cap M, f_{i}^{(M / K)}\right\}\right)\right\} .
$$

Lemma 5.28. The norm map $\widetilde{\mathcal{N}}_{L / M}: \widetilde{\mathbb{X}}(L / K)^{\times} \rightarrow \widetilde{\mathbb{X}}(M / K)^{\times}$introduced by (5.15) and (5.16) further satisfies

(i) $\tilde{\mathcal{N}}_{L / M}\left(Z_{L / K}\left(\left\{K_{i}, f_{i}^{(L / K)}\right\}\right)\right) \subseteq Z_{M / K}\left(\left\{K_{i} \cap M, f_{i}^{(M / K)}\right\}\right)$;

(ii) $\tilde{\mathcal{N}}_{L / M}\left(Y_{L / K}\right) \subseteq Y_{M / K}$.

Proof. Recall that $\widetilde{\mathcal{N}}_{L / M}: \widetilde{\mathbb{X}}(L / K)^{\times} \rightarrow \widetilde{\mathbb{X}}(M / K)^{\times}$is a continuous mapping.

(i) For any choice of $z^{(i)} \in Z_{i}^{(L / K)}$, the continuity of the multiplicative arrow $\widetilde{\mathcal{N}}_{L / M}$ : $\widetilde{\mathbb{X}}(L / K)^{\times} \rightarrow \widetilde{\mathbb{X}}(M / K)^{\times}$yields

$$
\widetilde{\mathcal{N}}_{L / M}\left(\prod_{i} z^{(i)}\right)=\prod_{i} \widetilde{\mathcal{N}}_{L / M}\left(z^{(i)}\right),
$$

where $\widetilde{\mathcal{N}}_{L / M}\left(z^{(i)}\right) \in Z_{i}^{(M / K)}$ by the commutative square (5.51).

(ii) Now let $y \in Y_{L / K}$. Then $y^{1-\varphi} \in Z_{L / K}\left(\left\{K_{i}, f_{i}^{(L / K)}\right\}\right)$. Hence, $\tilde{\mathcal{N}}_{L / M}\left(y^{1-\varphi}\right)=$ $\widetilde{\mathcal{N}}_{L / M}(y)^{1-\varphi} \in Z_{M / K}\left(\left\{K_{i} \cap M, f_{i}^{(M / K)}\right\}\right)$ by part (i), and the result follows.

Thus, the norm map $\widetilde{\mathcal{N}}_{L / M}: \widetilde{\mathbb{X}}(L / K)^{\times} \rightarrow \widetilde{\mathbb{X}}(M / K)^{\times}$defined by (5.16) induces a group homomorphism, which will again be called the Coleman norm map from $L$ to $M$,

$$
\widetilde{\mathcal{N}}_{L / M}^{\text {Coleman }}: U_{\widetilde{\mathbb{X}}(L / K)}^{\diamond} / Y_{L / K} \rightarrow U_{\widetilde{\mathbb{X}}(M / K)}^{\diamond} / Y_{M / K},
$$

and which is defined by

$$
\tilde{\mathcal{N}}_{L / M}^{\text {Coleman }}(\bar{U})=\tilde{\mathcal{N}}_{L / M}(U) . Y_{M / K}
$$

for every $U \in U_{\widetilde{\mathbb{X}}(L / K)}^{\diamond}$, where, as usual, $\bar{U}$ denotes the coset $U \cdot Y_{L / K}$ in $U_{\widetilde{\mathbb{X}}(L / K)}^{\diamond} / Y_{L / K}$.

Let

$$
\Phi_{M / K}^{(\varphi)}: \operatorname{Gal}(M / K) \rightarrow U_{\widetilde{\mathbb{X}}(M / K)}^{\diamond} / Y_{M / K}
$$

be the corresponding Fesenko reciprocity map defined for the extension $M / K$, where $Y_{M / K}=Y_{M / K}\left(\left\{K_{i} \cap M, f_{i}^{(M / K)}\right\}\right)$.

Theorem 5.29. For the Galois subextension $M / K$ of $L / K$, the square

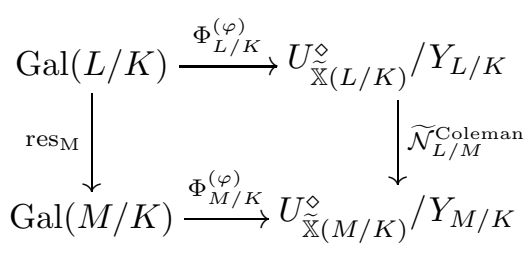


where the right vertical arrow

$$
\widetilde{\mathcal{N}}_{L / M}^{\text {Coleman }}: U_{\widetilde{\mathbb{X}}(L / K)}^{\ominus} / Y_{L / K} \rightarrow U_{\widetilde{\mathbb{X}}(M / K)}^{\ominus} / Y_{M / K}
$$

is the Coleman norm map from $L$ to $M$ defined by (5.52) and (5.53), is commutative.

Proof. It suffices to prove that the square

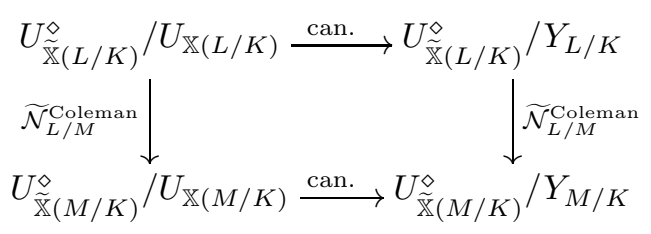

is commutative, which is obvious. Then pasting this square to the square (5.19), we obtain the diagram

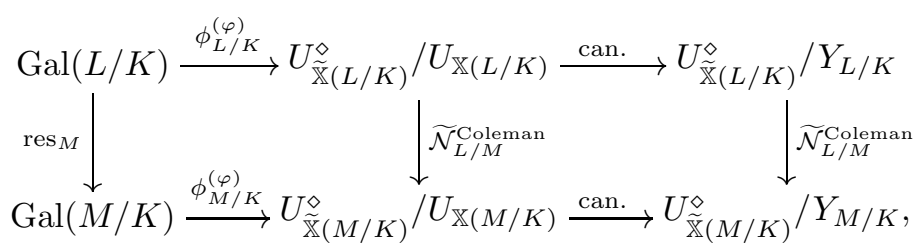

and the commutativity of the square (5.54) follows.

Let $F / K$ be a finite subextension of $L / K$. Then, since $F$ is compatible with $(K, \varphi)$ in the sense of [13, p. 89], we may fix the Lubin-Tate splitting over $F$ to be $\varphi_{F}=\varphi_{K}=\varphi$. Thus, there exists a chain of field extensions

$$
K \subseteq F \subseteq L \subseteq K_{\varphi} \subseteq F_{\varphi}
$$

where $L$ is a totally ramified APF Galois extension over $F$ by Lemma 3.3. Since $\boldsymbol{\mu}_{p}\left(K^{\mathrm{sep}}\right)=\boldsymbol{\mu}_{p}\left(F^{\mathrm{sep}}\right)$, the inclusion $\boldsymbol{\mu}_{p}\left(F^{\mathrm{sep}}\right) \subset F$ is satisfied. That is, the local field $F$ satisfies the condition given by (5.26).

Now, the basic ascending chain of subextensions in $L / K$ fixed in (5.27), with base changed to $F$,

$$
F=K_{o} F \subseteq K_{1} F \subseteq \cdots \subseteq K_{i} F \subseteq \cdots \subseteq L,
$$

is almost a basic ascending chain of subextensions in $L / F$, which follows by the iso-

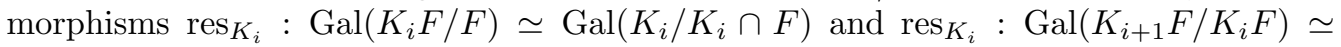
$\operatorname{Gal}\left(K_{i+1} / K_{i+1} \cap K_{i} F\right)$ for every $0 \leq i \in \mathbb{Z}$. Moreover, by the primitive element theorem, there exists $0 \leq i_{o} \in \mathbb{Z}$ such that $F \subseteq K_{i_{o}}$. If we choose the minimal $i_{o}$, the ascending chain (5.55) becomes

$$
F=K_{o} F \subseteq K_{1} F \subseteq \cdots \subseteq K_{i_{o}-1} F=K_{i_{o}} \subset K_{i_{o}+1} \subset \cdots \subset L .
$$

For each $1 \leq i \in \mathbb{Z}$, let $\sigma_{i}$ denote the element in $\operatorname{Gal}(\widetilde{L} / \widetilde{K})$ that satisfies

$$
\left\langle\left.\sigma_{i}\right|_{K_{i}}\right\rangle=\operatorname{Gal}\left(K_{i} / K_{i-1}\right) .
$$

Now, for $1 \leq i \in \mathbb{Z}$, we introduce elements $\sigma_{i}^{*}$ in $\operatorname{Gal}(\widetilde{L} / \widetilde{F})$ that satisfy

$$
\left\langle\left.\sigma_{i}^{*}\right|_{K_{i} F}\right\rangle=\operatorname{Gal}\left(K_{i} F / K_{i-1} F\right)
$$

as follows:

(i) for $i>i_{o}$ we define $\sigma_{i}^{*}=\sigma_{i}$; 
(ii) for $i \leq i_{o}$ we define

$$
\sigma_{i}^{*}=\left\{\begin{array}{lll}
\sigma_{i} & \text { if } & K_{i-1} F \subset K_{i} F, \\
\operatorname{id}_{K_{i} F} & \text { if } & K_{i-1} F=K_{i} F .
\end{array}\right.
$$

Then, clearly, for each $1 \leq i \in \mathbb{Z}$ the elements $\sigma_{i}^{*}$ of $\operatorname{Gal}(\widetilde{L} / \widetilde{F})$ satisfy

$$
\left\langle\left.\sigma_{i}^{*}\right|_{K_{i} F}\right\rangle=\operatorname{Gal}\left(K_{i} F / K_{i-1} F\right),
$$

and $\sigma_{i}^{*}=\sigma_{i}$ for almost all $i$. Moreover, for each $1 \leq k \in \mathbb{Z}$, the square

$$
\begin{aligned}
\operatorname{Gal}\left(K_{k+1} F / K_{k} F\right) \stackrel{\Xi_{K_{k+1} F / K_{k} F}}{\longrightarrow} & U_{\widetilde{K_{k+1}} F} / U_{\overparen{K_{k+1} F}}^{\sigma_{k+1}^{*}-1} \\
\operatorname{Gal}\left(K_{k+1} / K_{k}\right) \stackrel{\Xi_{K_{k+1} / K_{k}}}{\longrightarrow} & U_{\widetilde{K}_{k+1}} / \widetilde{N}_{\widetilde{K}_{k+1}^{*} F / K_{k+1}}^{\sigma_{k+1}-1}
\end{aligned}
$$

is commutative, because $\widetilde{N}_{K_{k+1} F / K_{k+1}}\left(\pi_{K_{k+1} F}\right)=\pi_{K_{k+1}}$ by the norm coherence of the Lubin-Tate labeling $(\pi_{K^{\prime}} \underbrace{K \subseteq K^{\prime} \subset K_{\varphi}}_{\left[K^{\prime}: K\right]<\infty}$. Hence,

$$
\widetilde{N}_{K_{k+1} F / K_{k+1}}^{*}\left(T_{k}^{(L / F)}\right)=T_{k}^{(L / K)} .
$$

Now, by Theorem [5.14, there exists an arrow

$$
h_{k}^{(L / F)}: \prod_{1 \leq i \leq k} U_{\widetilde{K_{k} F}}^{\sigma_{i}^{*}-1} \rightarrow\left(\prod_{1 \leq i \leq k+1} U_{\widetilde{K_{k+1} F}}^{\sigma_{i}^{*}-1}\right) / U_{\overparen{K_{k+1} F}}^{\sigma_{k+1}^{*}-1}
$$

that splits the exact sequence

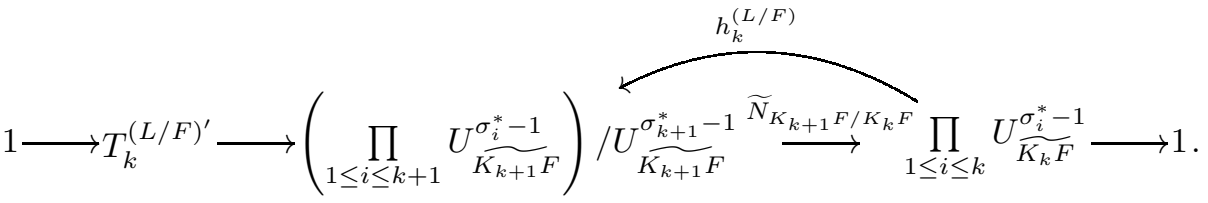

We choose an arrow

$$
h_{k}^{(L / K)}: \prod_{1 \leq i \leq k} U_{\widetilde{K_{k}}}^{\sigma_{i}-1} \rightarrow\left(\prod_{1 \leq i \leq k+1} U_{\widetilde{K_{k+1}}}^{\sigma_{i}-1}\right) / U_{\bar{K}_{k+1}}^{\sigma_{k+1}-1}
$$

that splits the exact sequence (5.47) in such a way that

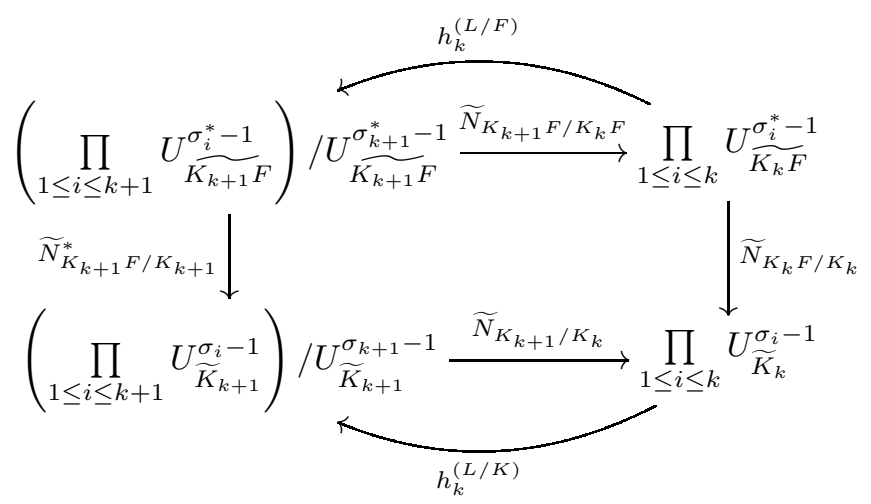


is a commutative square. The arrow (5.57) is constructed by following the same lines as in the construction of the arrow (5.44). For each $1 \leq k \in \mathbb{Z}$, consider any map

$$
g_{k}^{(L / K)}: \prod_{1 \leq i \leq k} U_{\widetilde{K}_{k}}^{\sigma_{i}-1} \rightarrow \prod_{1 \leq i \leq k+1} U_{\widetilde{K}_{k+1}}^{\sigma_{i}-1}
$$

that makes the following square commutative:

$$
\begin{aligned}
& \prod_{1 \leq i \leq k} U_{\widetilde{K_{k} F}}^{\sigma_{i}^{*}-1} \stackrel{g_{k}^{(L / F)}}{\longrightarrow} \prod_{1 \leq i \leq k+1} U_{\overline{K_{k+1}} F}^{\sigma_{i}^{*}-1} \\
& \widetilde{N}_{K_{k} F / K_{k}} \downarrow \downarrow \downarrow \widetilde{N}_{K_{k+1} F / K_{k+1}} \\
& \prod_{1 \leq i \leq k} U_{\widetilde{K}_{k}}^{\sigma_{i}-1} \quad g_{k}^{(L / K)} \cdots \cdots \prod_{1 \leq i \leq k+1} U_{\widetilde{K}}^{\sigma_{i}-1}
\end{aligned}
$$

Note that such a map satisfies $h_{k}^{(L / K)}=g_{k}^{(L / K)} \bmod U_{\widetilde{K}_{k+1}}^{\sigma_{k+1}-1}$. Indeed, by the commutative diagram (5.58), for any $w \in \prod_{1 \leq i \leq k} U_{\widetilde{K}_{k}}^{\sigma_{i}-1}$ there exists $v \in \prod_{1 \leq i \leq k} U_{\widetilde{K_{k} F}}^{\sigma_{i}^{*}-1}$ such that $w=\widetilde{N}_{K_{k} F / K_{k}}(v)$, and

$$
\begin{aligned}
h_{k}^{(L / K)}(w) & =h_{k}^{(L / K)}\left(\widetilde{N}_{K_{k} F / K_{k}}(v)\right) \\
& =\widetilde{N}_{K_{k+1} F / K_{k+1}}^{*}\left(h_{k}^{(L / F)}(v)\right) \\
& =\widetilde{N}_{K_{k+1} F / K_{k+1}}^{*}\left(g_{k}^{(L / F)}(v) \cdot U_{K_{k+1} F}^{\sigma_{k+1}^{*}-1}\right) \\
& =\widetilde{N}_{K_{k+1} F / K_{k+1}}\left(g_{k}^{(L / F)}(v)\right) \cdot U_{\widetilde{K}_{k+1}}^{\sigma_{k+1}-1},
\end{aligned}
$$

and by the commutativity of the diagram (5.60) we have

$$
\widetilde{N}_{K_{k+1} F / K_{k+1}}\left(g_{k}^{(L / F)}(v)\right)=g_{k}^{(L / K)}\left(\widetilde{N}_{K_{k} F / K_{k}}(v)\right)=g_{k}^{(L / K)}(w) .
$$

Thus, the relation

$$
h_{k}^{(L / K)}(w)=g_{k}^{(L / K)}(w) \cdot U_{\widetilde{K}_{k+1}}^{\sigma_{k+1}-1}
$$

follows for every $w \in \prod_{1 \leq i \leq k} U_{\widetilde{K}_{k}}^{\sigma_{i}-1}$.

Now, for each $1 \leq i \in \mathbb{Z}$, we introduce the map

$$
f_{i}^{(L / K)}: U_{\widetilde{K}_{i}}^{\sigma_{i}-1} \rightarrow U_{\widetilde{\mathbb{X}}(L / K)}
$$

by

$$
f_{i}^{(L / K)}(w)=\Lambda_{F / K}\left(f_{i}^{(L / F)}(v)\right),
$$

where $v \in U_{\widetilde{K_{i} F}}^{\sigma_{i}^{*}-1}$ is any element satisfying $\widetilde{N}_{K_{i} F / K_{i}}(v)=w \in U_{\widetilde{K}_{i}}^{\sigma_{i}-1}$. Note that if $v^{\prime} \in U_{\widetilde{K_{i} F}}^{\sigma_{i}^{*}-1}$ is such that $\widetilde{N}_{K_{i} F / K_{i}}\left(v^{\prime}\right)=w$, then $\Lambda_{F / K}\left(f_{i}^{(L / F)}(v)\right)=\Lambda_{F / K}\left(f_{i}^{(L / F)}\left(v^{\prime}\right)\right)$. In fact, there exists $u \in \operatorname{ker}\left(\widetilde{N}_{K_{i} F / K_{i}}\right)$ such that $v^{\prime}=v u$. Thus, we must verify that $\Lambda_{F / K}\left(f_{i}^{(L / F)}(v)\right)=\Lambda_{F / K}\left(f_{i}^{(L / F)}(v u)\right)$. That is, for each $1 \leq j \in \mathbb{Z}$, we must check the identity

$$
\operatorname{Pr}_{\widetilde{K}_{j}}\left(\Lambda_{F / K}\left(f_{i}^{(L / F)}(v)\right)\right)=\operatorname{Pr}_{\widetilde{K}_{j}}\left(\Lambda_{F / K}\left(f_{i}^{(L / F)}(v u)\right)\right) .
$$


Observe that for $j>i$ we have

$$
\begin{aligned}
\operatorname{Pr}_{\widetilde{K}_{j}}\left(\Lambda_{F / K}\left(f_{i}^{(L / F)}(v)\right)\right) & =\widetilde{N}_{K_{j} F / K_{j}}\left(\operatorname{Pr}_{\widetilde{K_{j} F}}\left(\Lambda_{F / K}\left(f_{i}^{(L / F)}(v)\right)\right)\right) \\
& =\widetilde{N}_{K_{j} F / K_{j}}\left(\operatorname{Pr}_{\widetilde{K_{j} F}}\left(f_{i}^{(L / F)}(v)\right)\right) \\
& =\widetilde{N}_{K_{j} F / K_{j}}\left(g_{j-1}^{(L / F)} \circ \cdots \circ g_{i}^{(L / F)}(v)\right) \\
& =g_{j-1}^{(L / K)} \circ \cdots \circ g_{i}^{(L / K)}\left(\widetilde{N}_{K_{i} F / K_{i}}(v)\right)
\end{aligned}
$$

because the square (5.60) is commutative. Now, the identity $\widetilde{N}_{K_{i} F / K_{i}}(v)=\widetilde{N}_{K_{i} F / K_{i}}(v u)$ implies (5.60). Thus, the map

$$
f_{i}^{(L / K)}: U_{\widetilde{K}_{i}}^{\sigma_{i}-1} \rightarrow U_{\widetilde{\mathbb{X}}(L / K)}
$$

is well defined. Moreover,

$$
\operatorname{Pr}_{\widetilde{K}_{j}} \circ f_{i}^{(L / K)}=\left.\left(g_{j-1}^{(L / K)} \circ \cdots \circ g_{i}^{(L / K)}\right)\right|_{U_{\widetilde{K}_{i}}^{\sigma_{i}-1}}
$$

for $j>i$. Indeed, for $w \in U_{\widetilde{K}_{i}}^{\sigma_{i}-1}$, there exists $v \in U_{\bar{K}_{i} F}^{\sigma_{i}^{*}-1}$ with $\widetilde{N}_{K_{i} F / K_{i}}(v)=w$, and $f_{i}^{(L / K)}(w)=\Lambda_{F / K}\left(f_{i}^{(L / F)}(v)\right)$. That is, the square

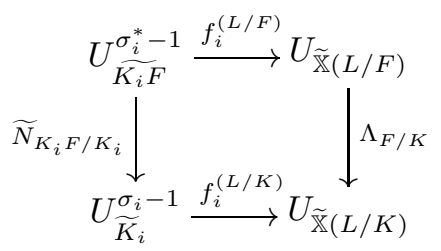

is commutative. Consequently, for $j>i$ we have

$$
\begin{aligned}
\operatorname{Pr}_{\widetilde{K}_{j}} \circ f_{i}^{(L / K)}(w) & =\operatorname{Pr}_{\widetilde{K}_{j}} \circ \Lambda_{F / K}\left(f_{i}^{(L / F)}(v)\right) \\
& =\widetilde{N}_{K_{j} F / K_{j}}\left(\operatorname{Pr}_{\widetilde{K_{j} F}} \circ f_{i}^{(L / F)}(v)\right) \\
& =\widetilde{N}_{K_{j} F / K_{j}}\left(\left(g_{j-1}^{(L / F)} \circ \cdots \circ g_{i}^{(L / F)}\right)(v)\right) \\
& =\left(g_{j-1}^{(L / K)} \circ \cdots \circ g_{i}^{(L / K)}\right)\left(\widetilde{N}_{K_{i} F / K_{i}}(v)\right),
\end{aligned}
$$

by the commutativity of the diagram (5.60), as desired.

For each $0<i \in \mathbb{Z}$, let

$$
Z_{i}^{(L / K)}=\operatorname{im}\left(f_{i}^{(L / K)}\right) .
$$

Then, by Lemma 5.15 or by [2, Lemma 4], for $z^{(i)} \in Z_{i}^{(L / K)}$ the product $\prod_{i} z^{(i)}$ converges to an element in $U_{\widetilde{\mathbb{X}}(L / K)}^{\diamond}$. Let

$$
Z_{L / K}\left(\left\{K_{i}, f_{i}^{(L / K)}\right\}\right)=\left\{\prod_{i} z^{(i)}: z^{(i)} \in Z_{i}^{(L / K)}\right\}
$$

which is a topological subgroup of $U_{\widetilde{\mathbb{X}}(L / K)}^{\diamond}$. We introduce the topological $\operatorname{Gal}(L / K)$ submodule $Y_{L / K}\left(\left\{K_{i}, f_{i}^{(L / K)}\right\}\right)=Y_{L / K}$ of $U_{\widetilde{\mathbb{X}}(L / K)}^{\diamond}$ by

$$
Y_{L / K}=\left\{y \in U_{\widetilde{\mathbb{X}}(L / K)}: y^{1-\varphi} \in Z_{L / K}\left(\left\{K_{i}, f_{i}^{(L / K)}\right\}\right)\right\} .
$$


Lemma 5.30. The continuous homomorphism $\Lambda_{F / K}: \widetilde{\mathbb{X}}(L / F)^{\times} \rightarrow \widetilde{\mathbb{X}}(L / K)^{\times}$introduced by (5.20) and (5.21) further satisfies

(i) $\Lambda_{F / K}\left(Z_{L / F}\left(\left\{K_{i} F, f_{i}^{(L / F)}\right\}\right)\right) \subseteq Z_{L / K}\left(\left\{K_{i}, f_{i}^{(L / K)}\right\}\right)$;

(ii) $\Lambda_{F / K}\left(Y_{L / F}\right) \subseteq Y_{L / K}$.

Proof. (i) For any choice of $z^{(i)} \in Z_{i}^{(L / F)}$, the continuity of the multiplicative arrow $\Lambda_{F / K}: \widetilde{\mathbb{X}}(L / F)^{\times} \rightarrow \widetilde{\mathbb{X}}(L / K)^{\times}$yields

$$
\Lambda_{F / K}\left(\prod_{i} z^{(i)}\right)=\prod_{i} \Lambda_{F / K}\left(z^{(i)}\right)
$$

where $\Lambda_{F / K}\left(z^{(i)}\right) \in Z_{i}^{(L / K)}$ by the commutative square (5.62).

(ii) Let $y \in Y_{L / F}$. Then $y^{1-\varphi} \in Z_{L / F}\left(\left\{K_{i} F, f_{i}^{(L / F)}\right\}\right)$ and $\Lambda_{F / K}\left(y^{1-\varphi}\right)=\Lambda_{F / K}(y)^{1-\varphi}$ is an element of $Z_{L / K}\left(\left\{K_{i}, f_{i}^{(L / K)}\right\}\right)$ by part (i). The result follows.

So, the homomorphism $\Lambda_{F / K}: \widetilde{\mathbb{X}}(L / F)^{\times} \rightarrow \widetilde{\mathbb{X}}(L / K)^{\times}$defined by (5.21) induces a group homomorphism

$$
\lambda_{F / K}: U_{\widetilde{\mathbb{X}}(L / F)}^{\diamond} / Y_{L / F} \rightarrow U_{\widetilde{\mathbb{X}}(L / K)}^{\diamond} / Y_{L / K}
$$

defined by

$$
\lambda_{F / K}(\bar{U})=\Lambda_{F / K}(U) . Y_{L / K},
$$

for every $U \in U_{\widetilde{\mathbb{X}}(L / F)}^{\diamond}$ (as usual, $\bar{U}$ denotes the coset $U . Y_{L / F}$ in $\left.U_{\widetilde{\mathbb{X}}(L / F)}^{\diamond} / Y_{L / F}\right)$.

Let

$$
\Phi_{L / F}^{(\varphi)}: \operatorname{Gal}(L / F) \rightarrow U_{\widetilde{\mathbb{X}}(L / F)}^{\diamond} / Y_{L / F}
$$

be the corresponding Fesenko reciprocity map defined for the extension $L / F$, where $Y_{L / F}=Y_{L / F}\left(\left\{K_{i} F, f_{i}^{(L / F)}\right\}\right)$.

Theorem 5.31. For the finite subextension $F / K$ of $L / K$, the square

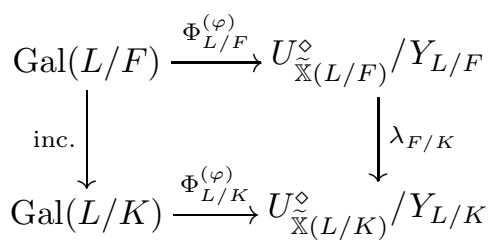

where the right vertical arrow $\lambda_{F / K}: U_{\widetilde{\mathbb{X}}(L / F)}^{\diamond} / Y_{L / F} \rightarrow U_{\widetilde{\mathbb{X}}(L / K)}^{\diamond} / Y_{L / K}$ is defined by (5.63) and (5.64), is commutative.

Proof. It suffices to prove that the square

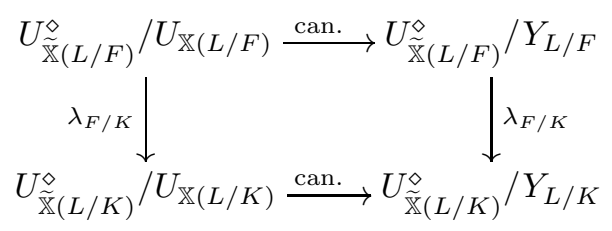


is commutative, which is obvious. Then, pasting this square to the square (5.24), we obtain the diagram

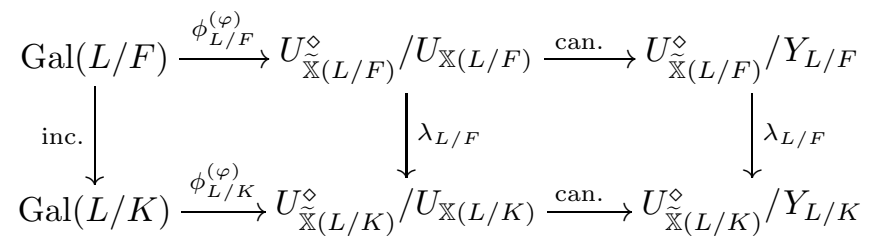

and the commutativity of the square (5.65) follows.

If, moreover, $L / K$ is a finite extension, then the square

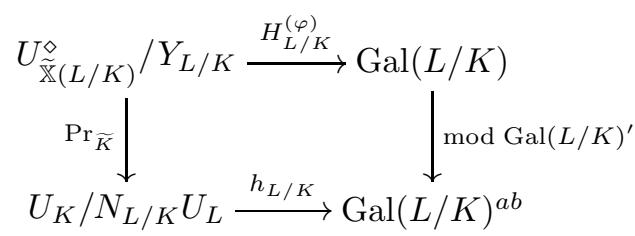

commutes. Thus, the inverse $H_{L / K}^{(\varphi)}=\left(\Phi_{L / K}^{(\varphi)}\right)^{-1}$ of the Fesenko reciprocity map $\Phi_{L / K}^{(\varphi)}$ defined for $L / K$ is a generalization of the Hazewinkel map for the totally ramified APF Galois subextensions $L / K$ of $K_{\varphi} / K$ under the assumption that the local field $K$ satisfies condition (5.26).

\section{ACKNOWLEDGMENTS}

The first author (K. I. I.) would like to thank the Institut de Mathématiques, "Théorie des Groupes, Représentations et Applications", Université Paris 7, Jussieu, Paris, and the School of Pure Mathematics of the Tata Institute of Fundamental Research, Mumbai, where some parts of this work were completed, for hospitality and support. The second author (E. S.) would like to thank TÜBITAK for a fellowship, and the School of Mathematics of the University of Nottingham, where some parts of this work have been started, for hospitality and support. Both authors would like to thank I. B. Fesenko for his interest and encouragement at all stages of this work.

\section{REFERENCES}

[1] I. B. Fesenko, Local reciprocity cycles, Invitation to Higher Local Fields (Münster, 1999) (I. B. Fesenko and M. Kurihara, eds.), Geom. Topol. Monogr., vol. 3, Geom. Topol. Publ., Coventry, 2000, pp. 293-298. MR1804942 (2001k:11239)

[2] _ Nonabelian local reciprocity maps, Class Field Theory - Its Centenary and Prospect (Tokyo, 1998) (K. Miyake, ed.), Adv. Stud. Pure Math., vol. 30, Math. Soc. Japan, Tokyo, 2001, pp. 63-78. MR1846451 (2002f:11177)

[3] - On the image of noncommutative local reciprocity map, Homology, Homotopy Appl. 7 (2005), 53-62. MR2200206 (2006m:11171)

[4] I. B. Fesenko and S. V. Vostokov, Local fields and their extensions. A constructive approach, Transl. Math. Monogr., vol. 121, Amer. Math. Soc., Providence, RI, 1993. MR.1218392 (94d:11095)

[5] J.-M. Fontaine and J.-P. Wintenberger, Le "corps des normes" de certaines extensions algébriques de corps locaux, C. R. Acad. Sci. Paris Sér. A-B 288 (1979), A367-A370. MR0526137 (80b:12015)

[6] _ Extensions algébriques et corps des normes des extensions APF des corps locaux, C. R. Acad. Sci. Paris Sér. A-B 288 (1979), A441-A444. MR0527692 (80h:12014)

[7] A. Gurevich, Description of Galois groups of local fields with the aid of power series, Ph. D. Thesis, Humboldt Univ., Berlin, 1998.

[8] M. Hazewinkel, Local class field theory is easy, Adv. Math. 18 (1975), 148-181. MR0389858 $(52: 10688)$ 
[9] K. I. Ikeda and M. Ikeda, Two lemmas on formal power series, Turkish J. Math. 23 (1999), 435-440. MR:1773548(2001f:13032)

[10] K. I. Ikeda and E. Serbest, Non-abelian local class field theory, Preprint.

[11] _ A remark on Sen's theory on Galois representations (in preparation).

[12] K. Iwasawa, Local class field theory, Oxford Univ. Press, Clarendon Press, New York, 1986. MR 0863740 (88b:11080)

[13] H. Koch and E. de Shalit, Metabelian local class field theory, J. Reine Angew. Math. 478 (1996), 85-106. MR 1409054 (97f:11095)

[14] F. Laubie, Une théorie du corps de classes local non abélien, Compositio Math. 143 (2007), 339362. MR2309990 (2008b:11124)

[15] J. Neukirch, Class field theory, Grundlehren Math. Wiss., Bd. 280, Springer-Verlag, Berlin, 1986. MR0819231 (87i:11005)

[16] J.-P. Serre, Corps locaux, 2-me éd., Publ. Inst. Math. Univ. Nancago, No. 8, Actualités Sci. Indust., No. 1296, Hermann, Paris, 1968. MR0354618 (50:7096)

[17] J.-P. Wintenberger, Le corps des normes de certaines extensions infinies de corps locaux; applications, Ann. Sci. École Norm. Sup. (4) 16 (1983), 59-89. MR0719763 (85e:11098)

Department of Mathematics, Istanbul Bilgi University, Kurtuluş Deresi Cad. No. 47, Dolapdere, 34440 BeyoĞLu, Istanbul, Turkey

E-mail address: ilhan@bilgi.edu.tr

Current address: Department of Mathematics, Yeditepe University, 26 Ağustos Yerleşimi, İnönü Mah., Kayışdağı Cad., 34755 Kadıköy, Istanbul, Turkey

E-mail address: ilhan.ikeda@yeditepe.edu.tr

Department of Mathematics, Atilim University, Kizilcaşar Köyü, Incek, 06836 Gölbaşi, AnKara, Turkey

E-mail address: eserbest@atilim.edu.tr

Received 20/AUG/2007

Originally published in English 